\title{
Effects of land use and sampling distance on water quality in tropical headwater springs (Pimenta creek, São Paulo State, Brazil)
}

\author{
Caio Vinicius Ferreira Marmontel ${ }^{\text {a }}$, Manuel Esteban Lucas-Borja ${ }^{\text {b,* }}$, \\ Valdemir Antonio Rodrigues ${ }^{a}$, Demetrio Antonio Zema ${ }^{c}$ \\ a Department of Forest Science, Univ. Estadual Paulista (UNESP), 18610-307 Botucatu, São Paulo State, Brazil \\ b Universidad de Castilla-La Mancha, Campus Albacete, 02071 Albacete, Spain \\ ${ }^{\text {c } M e d i t e r r a n e a ~ U n i v e r s i t y ~ o f ~ R e g g i o ~ C a l a b r i a, ~ D e p a r t m e n t ~ A G R A R I A, ~ l o c . ~ F e o ~ d i ~ V i t o, ~ I-89122 ~ R e g g i o ~ C a l a b r i a, ~ I t a l y ~}$
}

\section{H I G H L I G H T S}

- In tropical headwater streams information on water quality are in general scarce.

- Both land use and sampling distances influence water quality of headwaters.

- Springs with riparian vegetation show better conditions in the aquatic environment.

- In springs with pasture/agriculture and degraded vegetation water quality is worse.

- Aquatic environment of headwaters is very sensitive to changes in the environment.

\section{A R T I C L E I N F O}

\section{Article history:}

Received 30 October 2017

Received in revised form 1 December 2017

Accepted 2 December 2017

Available online 13 December 2017

Editor: D. Barcelo

\section{Keywords:}

Land use

Linear mixed model

Pasture

Riparian vegetation

Tropical forest

Water spring

\section{G R A P H I C A L A B S T R A C T}

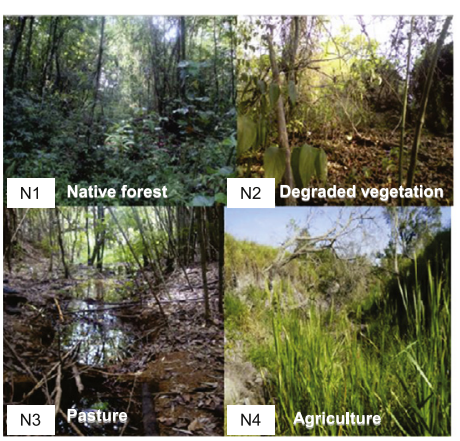

Effects of land use and sampling distance on

water quality in tropical headwater springs

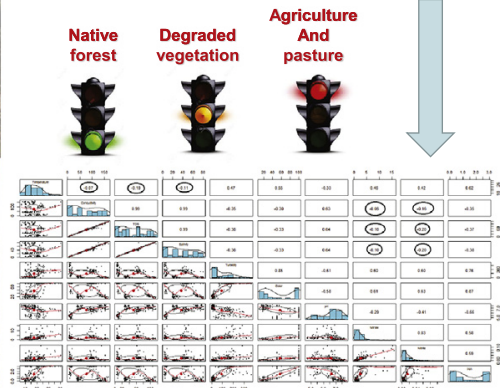

A B S T R A C T

The studies targeted to hydrology and water quality are scarce in tropical headwater streams. In these delicate ecosystems the comprehension of water quality can constitute a challenge, because the impact of land uses on stream dynamics is particularly severe in tropical areas. To fill this gap, an evaluation of water quality in a headwater streams (Pimenta creek, São Paulo State, Brazil) under tropical conditions was performed. The implementation of linear mixed models to water quality parameters allowed to know how and to what extent water flowing in these headwaters are influenced by: (i) the spatial variation of spring locations; (ii) the different land uses; and (iii) the state of conservation of the riparian vegetation. Both the land uses in the surroundings of water springs (native forest, degraded vegetation, agriculture and pasture) and the sampling points (exactly in the spring and 10,30 and $50 \mathrm{~m}$ downstream) were found to be factors able to explain water quality variability. Most of the analysed parameters, some of which strongly correlated each others (mainly electrical conductivity, Total Dissolved Solids and salinity, but also color, turbidity and iron concentrations), showed significant variations mainly due to the effects of the different land uses, but also to the distance from water spring. The instability of the water quality parameters in springs degraded from its headwater was also demonstrated. The water springs with developed riparian vegetation of natural forest (in a preserved or even disturbed conservation level) showed the best conditions in the aquatic environment (lower temperature, turbidity, color, nitrite and nitrate concentrations, neutral pH). Conversely, in the water springs with pasture or agricultural activities a general worsening of water quality was detected (worse

\footnotetext{
* Corresponding author.

E-mail address: ManuelEsteban.lucas@uclm.es (M.E. Lucas-Borja).
} 
turbidity, color, pH, nitrate and nitrate concentrations). Overall, the study has confirmed how much aquatic environment is sensitive to changes in the environment.

(C) 2017 Elsevier B.V. All rights reserved.

\section{Introduction}

Headwater streams (that is, the first- and second-order channels of a water course, Strahler, 1952), cumulatively constitute the great majority of channel length within a river network (Downing et al., 2012). Their importance within the ecology and health of a water course falls in the fact that headwater streams are the source of water, solutes, mineral sediment, and particulate organic matter (Schumm, 1977; Alexander et al., 2007; MacDonald and Coe, 2007; McClain and Naiman, 2008). These delicate ecosystems are strongly influenced by many disturbances factors, such as precipitation, morphology, land use, geology, vegetation, human impacts, which can affect the entire watershed supplied by their water flows (Wohl, 2017; Rodrigues et al., 2018). Furthermore, across diverse hydro-climatic regions, headwater streams tend to exhibit more spatial and temporal hydrologic variability than larger channels (Gomi et al., 2002; Richardson and Danehy, 2007), which strongly influences the river ecosystem. Given such stressing factors, it is necessary to pay attention to the physical, chemical, and biological functions of headwater streams and, in particular, to water quality. Recently, Wohl (2017) highlighted the importance of water chemistry analysis in headwater for at least two reasons: (i) headwater stream chemistry is highly influenced by upland flow paths and chemistry of incoming surface and ground waters; (ii) headwaters are the first line of defense against potential contaminants such as excess fine sediment or nutrients.

Unfortunately, the relatively small streams are currently rather ignored by legal protections (mostly extended to larger rivers) and are aggressively altered in connection with diverse land uses (Wohl, 2017), even though there has been a recent upsurge in interest in the restoration of riparian habitats, which is focusing attention on understanding and ameliorating such impacts (Bombino et al., 2007).

Water quality of headwater streams is important, because not only it is highly influenced by both upland flow paths and incoming surface and groundwaters, but also due to the fact that headwaters are the first line of defense against potential contaminants such as excess fine sediment or nutrients and the first receiving point for organic matter (Alexander et al., 2007). Also land use has significant impacts on river water quality with complex mechanisms, as demonstrated by several comparative studies (e.g. Wear et al., 1998; Amiri and Nakane, 2009; Ding et al., 2015). Although the significant impact of land use on stream water quality has been well documented (Johnson and Gage, 1997; Allen, 2004; Hurley and Mazumder, 2013; Bu et al., 2014; Ye et al., 2014; Kändler et al., 2017), further study on the complex association should be considered as much as possible (Yu et al., 2016). Therefore, it is important to carry out specific monitoring activities about the effects of land use on water quality specifically targeted to water springs of headwater streams.

Many different papers have dealt with monitoring and modeling of water quality at catchment-scale in several environments (e.g. Emmett et al., 1994; Ferrier et al., 2001; Baker, 2003; Ahearn et al., 2005; Shrestha and Kazama, 2007; Amiri and Nakane, 2009; Hurley and Mazumder, 2013; Bu et al., 2014; Ye et al., 2014; Viswanathan et al., 2015; Yu et al., 2016; Kändler et al., 2017). It has been highlighted that hydrology, light, temperature and water chemistry are controlled by regional factors such as geology, topography or climate (operating at spatial scales of catchments as well as ecoregions), and, in addition, that human land-use activities act to change both local and regional variables at an increasing rate (Bere and Tundisi, 2011). Therefore, it is evident that the analysis of water quality must be carried out by sitespecific studies.
However, the studies targeted to hydrology and water quality in tropical catchments are in general scarce (Fujieda et al., 1997); in addition, the comprehension of water quality response of a tropical catchment can constitute a challenge, because hydrological processes in these areas are difficult to assess (Hunke et al., 2015a, 2015b). Moreover, if we consider that the impact of land uses on stream water quality dynamics is particularly severe in tropical areas due to a more rapid mineralization of tropical soil organic matter and often, high erosion than in temperate zones (Spaans et al., 1990; Malmer and Grip, 1989; Hartemink et al., 2008), it is evident how important the evaluation of water quality and their variability factors under different land uses is in water spring of tropical headwater streams. In these contexts, the role of riparian vegetation tyipical of tropical forests must be also deepened. As a matter of fact, since riparian vegetation plays important hydrological and ecological functions in soil and natural resources protection, such as for instance stream water flow regularisation as well as conservation of river biodiversity and habitats (Tabacchi et al., 2000; Rocha et al., 2015), its role towards a greater stability of the physico-chemical characteristics of headwaters must be highlighted and enhanced.

Specific evaluations of water quality in Brazil are conducted at very few research stations, for example, clustered in the IBGE Reserve of the Federal District (Markewitz et al., 2006; Parron et al., 2010). Although more data are available from local and regional studies by local water managers or environmental protection agencies, they are not published in scientific journals and thus the impacts of land use on aquatic systems, that is, pollution from nutrients and pesticides, their in-stream processes, and their effects on aquatic habitats, are not well understood (Hunke et al., 2015a). Biome-specific water quality thresholds lack in Brazil (Hunke et al., 2015b), except for baselines for physical-chemical water parameters ranging from natural to very impacted conditions in the Cerrado area reported by Fonseca et al. (2014).

The objective of this work is the evaluation of water quality as influenced by the spatial variation of spring locations, the different land uses and state of conservation of the riparian vegetation in water springs of a headwater stream (São Paulo State, Brazil) typical of tropical conditions. More specifically, by applying linear mixed models the following questions are answered: (i) is water quality influenced by land use or distance from spring or both? (iii) to what extent water quality is influenced by these factors of change? (iii) are there any correlations among the water quality parameters? Identifying the spatial variability of land use impacts on water quality represents a significant challenge; addressing this issue is critical for assessing the potential risks of development and the cost-effectiveness of water management at the watershed scale (Ding et al., 2015).

\section{Materials and methods}

\subsection{Study site description}

The study was carried out in the headwater stream of Pimenta creek, a tributary of the Paraiso basin. The basin belongs to the São Manuel experimental farm (belonging to UNESP/FCA), in the central-western region of the state of São Paulo (Brazil) (Fig. 1). The basin of the Pimenta creek is located between the geographic coordinates $22^{\circ} 46^{\prime} 07^{\prime \prime} \mathrm{S}$ to $22^{\circ} 46^{\prime} 57^{\prime \prime} \mathrm{S}$ and $48^{\circ} 33^{\prime} 49^{\prime \prime} \mathrm{W}$ to $48^{\circ} 33^{\prime} 59^{\prime \prime} \mathrm{W}$ at an average altitude of $779 \mathrm{~m}$. It covers an area of 22.8 ha and is covered by pasture (57.5\%), native vegetation and bamboo (25.9\%), exotic vegetation (5.5\%), agriculture (10.1\%) and infrastructure (1.0\%); the main stream is $1620 \mathrm{~m}$ 

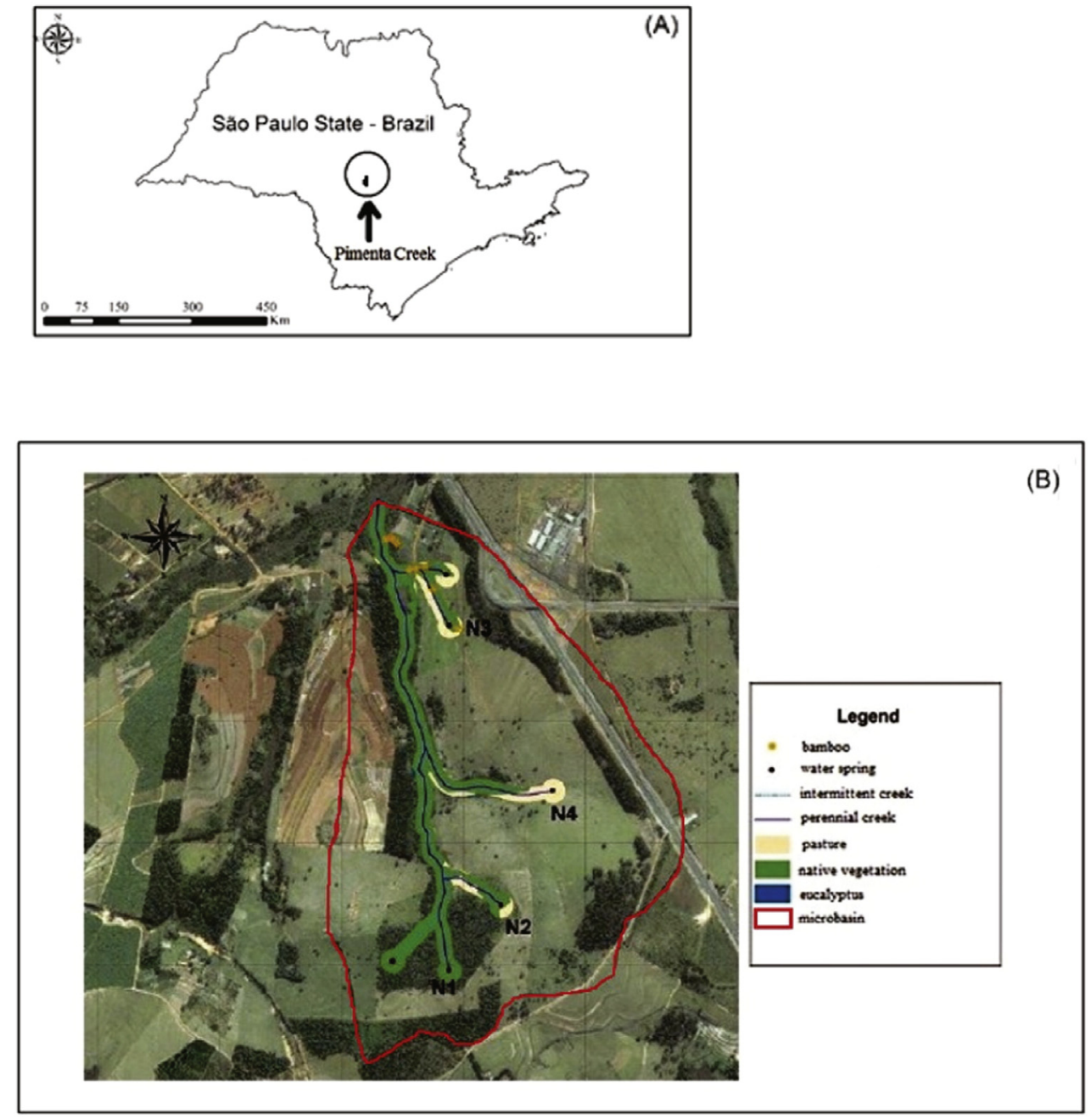

Fig. 1. Location (A) and aerial photo (B) of the Pimenta creek basin (São Paulo State, Brazil). (Source: adapted from Lima, 2003.)

long and its slope is of $2.6 \%$ up to $38.8 \%$. The climate of the region of São Manuel is of the type Cwa, hot temperate climate (mesothermic). The wetter and colder period falls in the spring-summer seasons and the water shortage with warmer temperatures in the autumn-winter seasons (Cunha and Martins, 2009) (Fig. 2). The floodplains of the water course show alluvial soils formed by sandy sediments (Lima, 2013). The soil of the basin, practically homogenous, is classified as Red-Dark Latosol, with sandy texture. It a soil in advanced stage of weathering, very evolved, as a result of notable transformations of the constitutive material.

\subsection{Sampling sites and water quality analyses}

In the studied basin four headwaters with as many water springs were identified (henceforth indicated as "N1", "N2", "N3" and "N4") (Fig. 3) and the land use characterized. The spring "N1" falls in a native tropical forest with a radius of $80 \mathrm{~m}$ around the source. Riparian vegetation has the physiognomic characteristics of the semidecidual seasonal forest and Cerrado. Spring "N2" is covered by secondary riparian forest developed after a wildfire occurred 40 years ago with some pasture on the left bank. The headwater of the spring "N3" is mainly pastured and in some zones bamboo (Bambusa sp.) cover was artificially established for erosion control; $30 \mathrm{~m}$ downstream of the headwater there is a narrow strip of riparian forest at its early stage. Around N3, domestic wastewater, treated and untreated, has been discharged for 50 years. In spring N4, pasture is cultivated around the spring with a cover of Brachiaria sp. In the rainy season, fertilizers and other chemical products

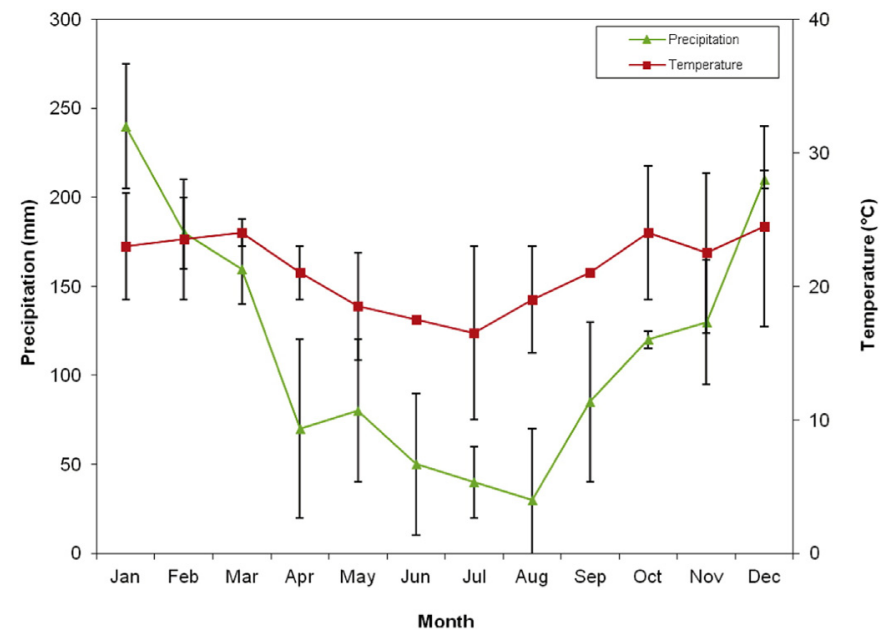

Fig. 2. Precipitation and temperature records (mean \pm std. dev., years 1971-2011) at São Manuel experimental farm (São Paulo State, Brazil). 


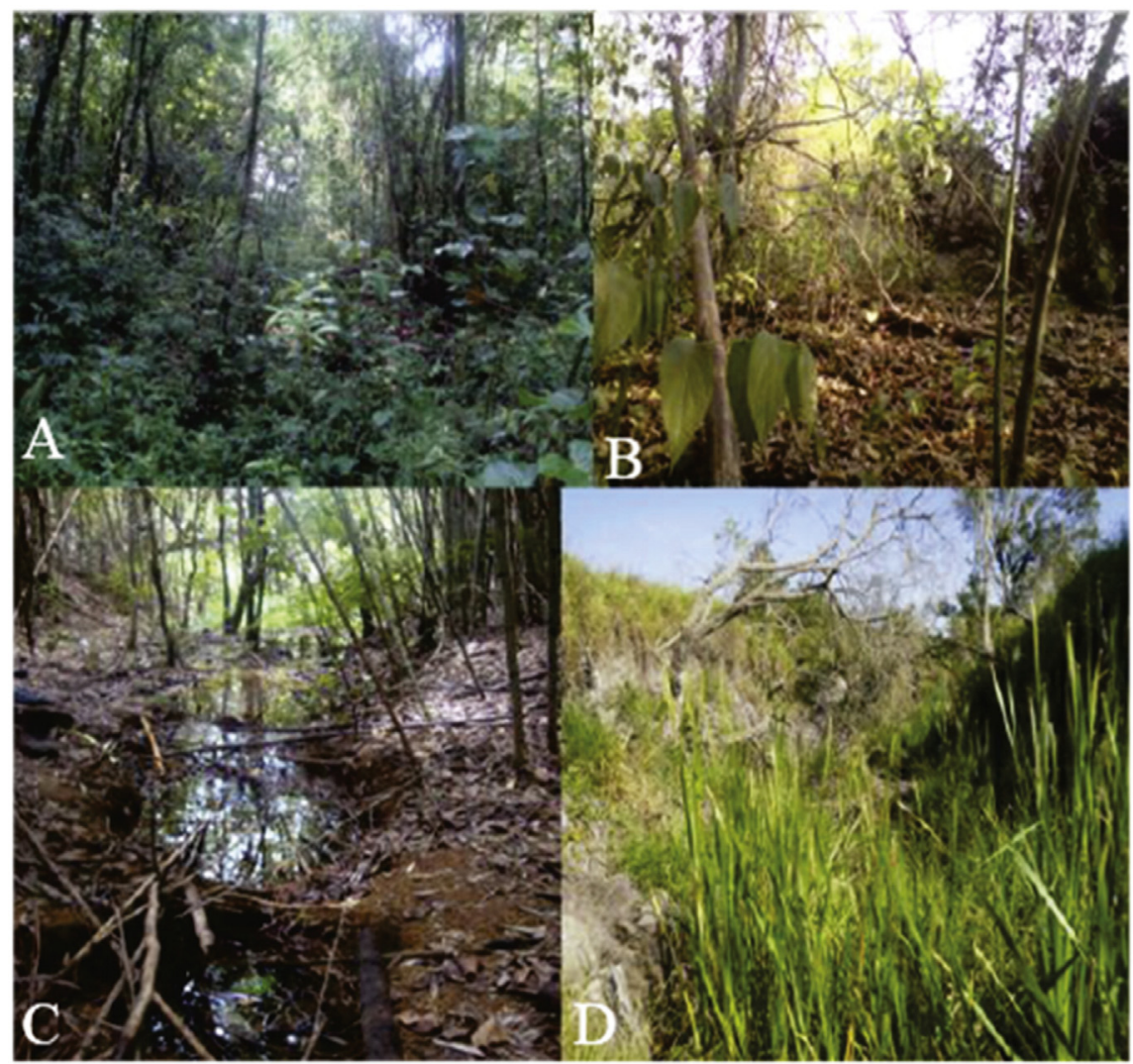

Fig. 3. Environment of the four water springs in the Pimenta creek (São Paulo State, Brazil). (Water springs A = "N1"; B = "N2"; C = "N3"; D = "N4").

are poured in the water course close to the spring, thus contributing for its degradation.

Stream discharges, measured in the channel immediately downstream of the four water springs, are higher between January and April (that is, during the wetter season, in which precipitation is concentrated) and lower during the drier period (particularly in August, when rainfall input is lower); on the average, the mean monthly values of stream discharge are quite similar among the four headwaters (from $0.17 \mathrm{~L} \mathrm{~s}^{-1}$ for $\mathrm{N} 2$ to $0.26 \mathrm{~L} \mathrm{~s}^{-1}$ for N3, Fig. 4).

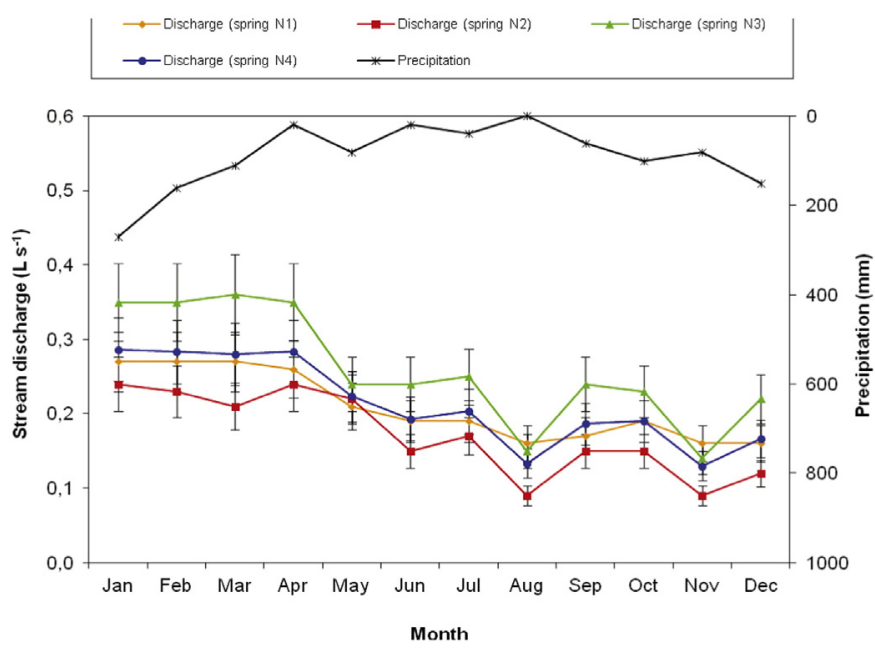

Fig. 4. Stream discharge and precipitation records (mean \pm std. dev., years 2012-2013) at water springs (N1, ..., N4) of Pimenta creek (São Paulo State, Brazil).
Close to these springs the riparian vegetation were characterized. Adopting the procedure described by Pinto (2003), the conservation level of the vegetation in the surroundings of each water spring was measured in four quadrants (up to a distance of about $10 \mathrm{~m}$ from the thalweg), with the right and left margin oriented along the flow direction of the main course. In relation to this conservation level, the springs were classified as "preserved", "disturbed" or "degraded", accordingly to the criteria reported in the Brazilian forest code (Federal Law no. 12.651/2012). In more detail, the riparian vegetation is considered: (i) "preserved", when it exists in the surroundings of $50 \mathrm{~m}$ from the spring without any signs of disturbance or degradation; (ii) "disturbed", if the spring does not show natural vegetation within a radius of $50 \mathrm{~m}$, but this space has a vegetation in good conditions and is covered partly by pasture or agriculture; (iii) "degraded", if the spring is subject to a high degree of disturbance, compacted soil, scarce and eroded vegetation. Therefore, the vegetation of springs "N1" and "N2" is "preserved forest" and "disturbed forest", respectively. Spring "N3" is a "degraded pasture", while vegetation of "N4" is classified as "degraded and agricultural").

In order to evaluate water quality, samples of water were collected systematically at four points for each of the four springs, with four measurements for each sampling point. In more detail, the first sampling point (henceforth "P1") was located exactly at spring source. The other samples were collected 10,30 and $50 \mathrm{~m}$ downstream of the water spring (indicated as "P2", "P3" and "P4", respectively); of course, each sampling point relates to a different distance from the source. Samples were collected throughout one year (from August 2012 to July 2013), distributed in monthly surveys.

In our study a limited but representative set of water quality parameters was selected. We excluded some measurements such as the concentrations of some cations $\left(\mathrm{Ca}^{++}, \mathrm{Mg}^{++}, \mathrm{K}^{+}\right.$and $\left.\mathrm{Na}^{+}\right)$and anions $\left(\mathrm{HCO}_{3}^{-}, \mathrm{SO}_{4}^{-}, \mathrm{Cl}^{-}\right)$, since water pollution by these elements/compounds was not suspected in the analysed springs, lacking in their surroundings 
mineral fertiliser use (containing some of the aforementioned cations) and industrial facilities (which may contaminate spring water with the anions above, beside heavy metals).

As regards the water quality parameters selected, the following determinations were made in situ:

- Electrical conductivity $\left[\mu \mathrm{S} \mathrm{cm}^{-1}\right]$, total dissolved solids (TDS, $\left.\left[\mathrm{mg} \mathrm{L}^{-1}\right]\right)$ and salinity [ $\left.\mathrm{mg} \mathrm{L}^{-1}\right]$, using the portable multimeters Extech PH 100 and EC 400;

- Temperature $\left[{ }^{\circ} \mathrm{C}\right]$ and $\mathrm{pH}[-]$ by a $\mathrm{pH}-$ meter (Extech $\mathrm{PH} 100$ ).

At the Water Quality Laboratory of the Department of Rural Engineering at Campus Botucatu of the São Paulo State University, the following water parameters were determined:

- Color [mg L $\mathrm{L}^{-1} \mathrm{Pt}$ ], by the colorimeter Aqua-Tester 611-A;

- Turbidity ([FAU], according to ISO Method 7027, attenuated radiation), nitrate $\left[\mathrm{mg} \mathrm{L}^{-1}\right]$, nitrite $\left[\mathrm{mg} \mathrm{L}^{-1}\right]$ and iron $\left[\mathrm{mg} \mathrm{L}^{-1}\right]$, by the digital spectrophotometer Hach Model DR2010 were measured. Phosphate concentration was not analysed in addition to nitrogen compounds, because the fertiliser used in the agricultural activities surrounding the analysed water springs is only animal manure, which, as well known, is rich in nitrogen and poor in phosphorous.

As reference limits for water quality evaluation and comparison, the standards issued by United States Environmental Protection Agency (USEPA) were adopted.

\subsection{Statistical analysis}

The water quality parameters were transformed to square root to fit the equity of variance and normal distribution, then the descriptive statistics for each variable were calculated. Subsequently, three Linear Mixed Models (henceforth indicated as "LMM ${ }_{s} \cdot d$ ", "LMM" and "LMM ${ }_{d}$ ") were applied to analyse whether there are any correlations (and their significance level) between the water quality parameters and: (i) spring characteristics (land use and conservation level of riparian vegetation) and distance from water spring (for $\mathrm{LMM}_{\mathrm{s} \cdot \mathrm{d}}$ ); (ii) only spring characteristics (for $\mathrm{LMM}_{\mathrm{s}}$ ); (iii) only distance from water spring $\left(\mathrm{LMM}_{\mathrm{d}}\right)$.

In order to find out which one of the three tested models best fits the data, the likelihood ratio test and the Akaike criterion were used. The results of the analyses performed for the three models were compared through the tables of analysis of variance. After the best LMM was defined, the Tukey test (at p-level $<0.05$ ) was applied to compare water quality parameters between the sampling points of each source and the correlation analyses were performed using the Pearson method (Viswanathan et al., 2015).

\section{Results}

In general, all the measured parameters of water quality were under the criteria suggested by USEPA, except for iron concentration (in our study in the range $0.5-2.6 \mathrm{mg} \mathrm{L}^{-1}$ on the average against a limit of $0.3-1.0 \mathrm{mg} \mathrm{L}^{-1}$ reported by USEPA); this leads to consider the water quality of the analysed spring as good.

\subsection{Water quality variations among water springs}

Among the analysed water springs, "N1" showed the lowest mean temperature $\left(19.3{ }^{\circ} \mathrm{C} \pm 1.5\right)$, turbidity (12.6 FAU \pm 6.1 ), nitrite ( $\left.0.005 \mathrm{mg} \mathrm{L}^{-1} \pm 0.003\right)$ and iron $\left(0.3 \mathrm{mg} \mathrm{L}^{-1} \pm 0.3\right)$, but the highest electrical conductivity ( $143.7 \mu \mathrm{S} \mathrm{cm}^{-1} \pm 12.1$ ), Total Dissolved Solids (100.7 $\left.\mathrm{mg} \mathrm{L}^{-1} \pm 8.3\right)$, salinity (71.7 $\left.\mathrm{mg} \mathrm{L}^{-1} \pm 5.7\right)$ and $\mathrm{pH}(7.3 \pm$
$0.2)$. The spring "N2" had the lowest mean color $\left(22.6 \mathrm{mg} \mathrm{L}^{-1} \mathrm{Pt} \pm\right.$ $7.5)$ and nitrate $\left(0.8 \mathrm{mg} \mathrm{L}^{-1} \pm 0.3\right)$. For "N3" the lowest mean electrical conductivity ( $\left.11.9 \mu \mathrm{S} \mathrm{cm}^{-1} \pm 3.6\right)$, TDS ( $\left.8.3 \mathrm{mg} \mathrm{L}^{-1} \pm 2.5\right)$, salinity (5.8 $\left.\mathrm{mg} \mathrm{L}^{-1} \pm 1.7\right)$ and $\mathrm{pH}(5.4 \pm 0.3)$ together with the highest mean turbidity (173.3 FAU \pm 78.1$)$ and nitrite $\left(0.1 \mathrm{mg} \mathrm{L}^{-1} \pm 0.02\right)$ were measured. "N4" presented the highest mean temperature (23.1 ${ }^{\circ} \mathrm{C} \pm 3$ ), color (95.3 $\left.\mathrm{mg} \mathrm{L}^{-1} \mathrm{Pt} \pm 10.8\right)$, nitrate $\left(4.8 \mathrm{mg} \mathrm{L}^{-1} \pm 2.7\right)$ and iron (2.6 $\left.\mathrm{mg} \mathrm{L}^{-1} \pm 0.6\right)$ (Table 1).

\subsection{Comparison between linear mixed models}

Table 2 reports the comparisons between the three linear mixed models (LMM) tested for analyzing the water quality parameters in the Pimenta creek. The differences between $\mathrm{LMM}_{\mathrm{s} \cdot \mathrm{d}}$ (based on spring characteristics and distance from the water spring) and $\mathrm{LMM}_{\mathrm{d}}$ (based only on distance) were significant for all the studied parameters. $\mathrm{LMM}_{\mathrm{s} \cdot \mathrm{d}}$ significantly differed from $\mathrm{LMM}_{\mathrm{s}}$ (based on spring characteristics) only for two parameters (temperature and iron concentration), while $\mathrm{LMM}_{\mathrm{s}}$ and $\mathrm{LMM}_{\mathrm{d}}$ (this latter based only on distance) gave practically the same statistical values (Table 2 ).

\subsection{Spatial variations of water quality in water springs}

The spatial differences in temperature and iron concentrations between the sampling points of each spring were not significant ( $p$-level $>0.9$ ) (Table 3). Conversely, it was observed that in some points the electrical conductivity, Total Dissolved Solids, salinity, turbidity, color, $\mathrm{pH}$, nitrate and nitrite were significantly influenced by the distance of water collection point from the spring. $>90 \%$ of these differences were detected between "P1" and the other sampling points ("P2", "P3" and "P4"). The parameter pH was found to have the highest spatial variability among the sampling points (Table 3 ).

More specifically, for spring "N1" (preserved riparian forest), only $\mathrm{pH}$ had significant differences between the sampling points, except for the couples of points "P1"-"P2" and "P3"-"P4". Water sampled at spring "N2" (covered by natural forest in a disturbed state) had more significant difference in pH between points "P1" and "P3"-"P4" as well as "P2" and "P4" (Table 3). The spring "N3" (pasture with degraded vegetation) showed significant differences between the sampling point "P1" and the other points ("P2", "P3" and "P4") mainly for the Total Dissolved Solids, turbidity and color and in some couples of sampling points for $\mathrm{pH}$, nitrate, nitrite and electrical conductivity). Finally, in the spring "N4" (with degraded vegetation as "N3", but agricultural) less significant differences (mainly in electrical conductivity, Total Dissolved Solids and salinity) were detected between the sampling point "P1" and the some other points at a distance from "P1" (Table 3).

\subsection{Correlations among water quality parameters}

As expected, electrical conductivity, Total Dissolved Solids and salinity were strongly correlated each another $(r>0.99)$ and this suggests that they express very similar water quality parameters and processes. All these parameters were not significantly correlated with temperature, nitrate and nitrite $(r<0.20)$. Finally, color has a very strong correlation with turbidity $(\mathrm{r}=0.85)$ and iron $(\mathrm{r}=0.87)$ and these latter parameters are also noticeably correlated each other $(r=0.76)$ (Fig. 5). Also Rodrigues et al. (2018) found a high correlation between iron and turbidity in the same tropical environment.

\section{Discussions}

Tropical catchments, such as the focus of this investigation, are precisely characterized by strong seasonality of climate with pronounced wet and dry seasons (Perez Hernandez and Lopez, 1998); moreover, also the generation of surface runoff, which influences water quality parameters, is a seasonal phenomenon, largely affected by land use and 
Table 1

Descriptive statistics of water quality parameters of water spring in the Pimenta creek (São Paulo State, Brazil).

\begin{tabular}{|c|c|c|c|c|c|c|c|c|c|c|c|}
\hline \multirow[t]{2}{*}{ Water spring sampling point } & Mean & Maximum & Minimum & Std. Dev. & CV & Water spring sampling point & Mean & Maximum & Minimum & \multirow[t]{2}{*}{ Std. Dev. } & \multirow[t]{2}{*}{$\mathrm{CV}$} \\
\hline & \multicolumn{6}{|c|}{ Temperature $\left({ }^{\circ} \mathrm{C}\right)$} & \multicolumn{3}{|c|}{ Electrical conductivity $\left(\mu \mathrm{Sm}^{-1}\right)$} & & \\
\hline N1P1 & 19,2 & 21,3 & 16,6 & 1,6 & 8,4 & N1P1 & 147,3 & 165,7 & 125,4 & 13,7 & 9,3 \\
\hline N1P2 & 19,3 & 21,3 & 16,7 & 1,5 & 7,8 & N1P2 & 140,0 & 154,3 & 121,3 & 11,4 & 8,2 \\
\hline N1P3 & 19,3 & 21,0 & 16,8 & 1,5 & 7,6 & N1P3 & 142,4 & 155,3 & 121,5 & 11,8 & 8,3 \\
\hline N1P4 & 19,3 & 21,4 & 16,8 & 1,5 & 7,9 & $\mathrm{~N} 1 \mathrm{P} 4$ & 145,1 & 161,6 & 129,0 & 11,3 & 7,8 \\
\hline Mean & 19,3 & 21,3 & 16,7 & 1,5 & 7,9 & Mean & 143,7 & 159,2 & 124,3 & 12,1 & 8,4 \\
\hline N2P1 & 20,0 & 23,2 & 17,2 & 1,8 & 8,8 & N2P1 & 49,3 & 67,9 & 41,0 & 8,1 & 16,4 \\
\hline N2P2 & 19,9 & 22,8 & 17,3 & 1,7 & 8,4 & $\mathrm{~N} 2 \mathrm{P} 2$ & 44,8 & 56,5 & 37,9 & 6,5 & 14,5 \\
\hline N2P3 & 19,8 & 22,4 & 17,3 & 1,6 & 8,3 & $\mathrm{~N} 2 \mathrm{P} 3$ & 44,3 & 59,9 & 38,1 & 6,7 & 15,1 \\
\hline N2P4 & 19,7 & 22,3 & 17,3 & 1,7 & 8,7 & $\mathrm{~N} 2 \mathrm{P} 4$ & 45,3 & 55,5 & 39,0 & 6,2 & 13,6 \\
\hline Mean & 19,9 & 22,7 & 17,3 & 1,7 & 8,6 & Mean & 45,9 & 60,0 & 39,0 & 6,9 & 14,9 \\
\hline N3P1 & 21,4 & 24,5 & 18,2 & 2,0 & 9,3 & N3P1 & 14,6 & 19,7 & 11,6 & 2,2 & 14,7 \\
\hline N3P2 & 21,8 & 24,7 & 18,2 & 1,9 & 8,9 & N3P2 & 11,5 & 18,5 & 5,0 & 4,2 & 36,2 \\
\hline N3P3 & 21,7 & 25,6 & 18,2 & 2,1 & 9,8 & N3P3 & 10,5 & 19,9 & 6,1 & 4,7 & 44,5 \\
\hline N3P4 & 21,6 & 25,0 & 18,1 & 2,2 & 10,1 & N3P4 & 10,9 & 19,9 & 7,1 & 3,4 & 31,4 \\
\hline Mean & 21,6 & 25,0 & 18,2 & 2,1 & 9,5 & Mean & 11,9 & 19,5 & 7,5 & 3,6 & 31,7 \\
\hline N4P1 & 23,5 & 30,2 & 18,3 & 3,6 & 15,3 & N4P1 & 111,3 & 153,3 & 93,0 & 16,9 & 15,1 \\
\hline N4P2 & 23,1 & 28,3 & 18,4 & 3,0 & 13,1 & N4P2 & 98,6 & 116,0 & 80,0 & 10,3 & 10,4 \\
\hline N4P3 & 23,1 & 28,1 & 18,7 & 2,8 & 12,1 & N4P3 & 100,5 & 136,7 & 82,1 & 15,2 & 15,1 \\
\hline N4P4 & 22,7 & 27,3 & 18,0 & 2,7 & 12,0 & N4P4 & 93,9 & 106,3 & 81,3 & 7,9 & 8,4 \\
\hline \multirow[t]{2}{*}{ Mean } & 23,1 & 28,5 & 18,4 & 3,0 & 13,1 & Mean & 101,1 & 128,1 & 84,1 & 12,5 & 12,3 \\
\hline & \multicolumn{6}{|c|}{ Total dissolved solids $\left(\mathrm{mg} \mathrm{L}^{-1}\right)$} & Salinity & $\left(\mathrm{mg} \mathrm{L}^{-1}\right)$ & & & \\
\hline N1P1 & 103,6 & 115,9 & 89,7 & 9,2 & 8,9 & N1P1 & 73,0 & 81,2 & 64,1 & 5,9 & 8,0 \\
\hline N1P2 & 97,6 & 108,0 & 84,6 & 8,2 & 8,4 & N1P2 & 69,7 & 77,3 & 60,5 & 5,9 & 8,4 \\
\hline N1P3 & 99,3 & 108,9 & 85,4 & 7,7 & 7,7 & N1P3 & 71,1 & 77,8 & 61,5 & 5,5 & 7,7 \\
\hline N1P4 & 102,2 & 113,4 & 91,4 & 8,1 & 7,9 & N1P4 & 72,9 & 80,6 & 65,3 & 5,8 & 7,9 \\
\hline Mean & 100,7 & 111,6 & 87,8 & 8,3 & 8,2 & Mean & 71,7 & 79,2 & 62,9 & 5,7 & 8,0 \\
\hline N2P1 & 34,5 & 47,5 & 28,9 & 5,8 & 16,7 & N2P1 & 24,5 & 34,0 & 20,6 & 4,1 & 16,5 \\
\hline N2P2 & 31,2 & 39,5 & 26,5 & 4,3 & 13,9 & N2P2 & 22,5 & 29,1 & 18,9 & 3,3 & 14,8 \\
\hline N2P3 & 30,8 & 39,4 & 26,9 & 4,4 & 14,2 & N2P3 & 22,0 & 28,0 & 19,1 & 3,0 & 13,8 \\
\hline N2P4 & 31,4 & 39,4 & 25,3 & 4,8 & 15,3 & N2P4 & 22,6 & 28,2 & 19,3 & 3,4 & 14,8 \\
\hline Mean & 32,0 & 41,5 & 26,9 & 4,8 & 15,0 & Mean & 22,9 & 29,8 & 19,5 & 3,4 & 15,0 \\
\hline N3P1 & 10,4 & 13,9 & 8,0 & 1,6 & 15,2 & N3P1 & 7,1 & 8,3 & 5,1 & 0,9 & 12,4 \\
\hline N3P2 & 8,3 & 16,2 & 3,5 & 3,7 & 45,1 & N3P2 & 5,8 & 10,1 & 2,6 & 2,6 & 43,9 \\
\hline N3P3 & 7,1 & 12,6 & 4,5 & 2,6 & 36,4 & N3P3 & 5,2 & 9,7 & 3,0 & 2,2 & 41,4 \\
\hline N3P4 & 7,2 & 10,9 & 4,5 & 1,9 & 26,0 & N3P4 & 5,1 & 7,5 & 3,1 & 1,4 & 27,0 \\
\hline Mean & 8,3 & 13,4 & 5,1 & 2,5 & 30,7 & Mean & 5,8 & 8,9 & 3,5 & 1,7 & 31,2 \\
\hline N4P1 & 73,4 & 106,5 & 60,9 & 13,0 & 17,7 & N4P1 & 54,5 & 80,1 & 35,1 & 13,3 & 24,4 \\
\hline N4P2 & 67,5 & 80,4 & 55,2 & 7,4 & 11,0 & N4P2 & 47,0 & 57,3 & 39,3 & 5,2 & 11,0 \\
\hline N4P3 & 63,5 & 70,1 & 56,2 & 3,6 & 5,6 & N4P3 & 44,8 & 50,8 & 39,8 & 3,0 & 6,6 \\
\hline N4P4 & 63,8 & 70,4 & 56,8 & 4,3 & 6,7 & N4P4 & 43,9 & 50,3 & 38,1 & 4,1 & 9,3 \\
\hline Mean & 67,0 & 81,9 & 57,3 & 7,1 & 10,2 & Mean & 47,5 & 59,6 & 38,1 & 6,4 & 12,8 \\
\hline & Turbidi & ty (FAU) & & & & & Color ( & $\left.\mathrm{ng} \mathrm{L} \mathrm{L}^{-1} \mathrm{Pt}\right)$ & & & \\
\hline N1P1 & 12,3 & 23,0 & 2,0 & 6,3 & 51,3 & N1P1 & 19,4 & 35,0 & 10,0 & 8,9 & 46,1 \\
\hline N1P2 & 14,2 & 22,0 & 4,0 & 5,1 & 36,0 & N1P2 & 25,4 & 40,0 & 17,5 & 7,1 & 28,1 \\
\hline N1P3 & 12,3 & 25,0 & 0,0 & 8,6 & 69,3 & N1P3 & 24,6 & 40,0 & 15,0 & 7,8 & 31,5 \\
\hline N1P4 & 11,6 & 21,0 & 3,0 & 4,4 & 37,7 & N1P4 & 25,8 & 55,0 & 15,0 & 11,0 & 42,5 \\
\hline Mean & 12,6 & 22,8 & 2,3 & 6,1 & 48,6 & Mean & 23,8 & 42,5 & 14,4 & 8,7 & 37,1 \\
\hline N2P1 & 11,9 & 34,0 & 0,0 & 10,3 & 86,6 & N2P1 & 19,4 & 30,0 & 10,0 & 5,8 & 29,7 \\
\hline N2P2 & 18,8 & 56,0 & 0,0 & 15,5 & 82,7 & $\mathrm{~N} 2 \mathrm{P} 2$ & 21,9 & 35,0 & 10,0 & 8,9 & 40,5 \\
\hline N2P3 & 16,5 & 46,0 & 1,0 & 11,4 & 68,8 & $\mathrm{~N} 2 \mathrm{P} 3$ & 22,9 & 35,0 & 15,0 & 6,6 & 28,6 \\
\hline $\mathrm{N} 2 \mathrm{P} 4$ & 16,3 & 44,0 & 2,0 & 12,6 & 76,9 & N2P4 & 26,3 & 50,0 & 17,5 & 8,8 & 33,4 \\
\hline Mean & 15,9 & 45,0 & 0,8 & 12,4 & 78,8 & Mean & 22,6 & 37,5 & 13,1 & 7,5 & 33,0 \\
\hline N3P1 & 70,9 & 119,0 & 20,0 & 33,3 & 46,9 & N3P1 & 63,3 & 100,0 & 40,0 & 24,6 & 38,9 \\
\hline N3P2 & 219,2 & 362,0 & 38,0 & 99,3 & 45,3 & N3P2 & 91,7 & 100,0 & 50,0 & 19,5 & 21,2 \\
\hline N3P3 & 205,3 & 366,0 & 60,0 & 101,5 & 49,5 & N3P3 & 96,3 & 100,0 & 55,0 & 13,0 & 13,5 \\
\hline N3P4 & 197,9 & 320,0 & 71,0 & 78,4 & 39,6 & N3P4 & 95,0 & 100,0 & 60,0 & 12,4 & 13,1 \\
\hline Mean & 173,3 & 291,8 & 47,3 & 78,1 & 45,3 & Mean & 86,6 & 100,0 & 51,3 & 17,4 & 21,7 \\
\hline N4P1 & 144,0 & 191,0 & 91,0 & 29,9 & 20,8 & N4P1 & 94,2 & 100,0 & 60,0 & 12,4 & 13,2 \\
\hline N4P2 & 155,4 & 230,0 & 100,0 & 37,0 & 23,8 & N4P2 & 92,9 & 100,0 & 60,0 & 13,6 & 14,6 \\
\hline N4P3 & 176,1 & 284,0 & 75,0 & 57,4 & 32,6 & N4P3 & 95,8 & 100,0 & 60,0 & 11,7 & 12,2 \\
\hline N4P4 & 161,9 & 256,0 & 100,0 & 40,3 & 24,9 & N4P4 & 98,3 & 100,0 & 80,0 & 5,8 & 5,9 \\
\hline Mean & 159,4 & 240,3 & 91,5 & 41,2 & 25,5 & Mean & 95,3 & 100,0 & 65,0 & 10,8 & 11,4 \\
\hline & $\mathrm{pH}(-$ & & & & & & Nitrate & $\left(\mathrm{mg} \mathrm{L}^{-1}\right)$ & & & \\
\hline N1P1 & 7,0 & 7,4 & 6,7 & 0,2 & 3,2 & N1P1 & 1,2 & 1,9 & 0,3 & 0,5 & 42,7 \\
\hline N1P2 & 7,1 & 7,6 & 6,8 & 0,2 & 2,8 & N1P2 & 1,1 & 1,9 & 0,4 & 0,5 & 46,7 \\
\hline N1P3 & 7,5 & 7,9 & 6,9 & 0,3 & 3,7 & N1P3 & 1,1 & 1,8 & 0,1 & 0,5 & 43,4 \\
\hline N1P4 & 7,5 & 7,7 & 7,0 & 0,2 & 2,5 & N1P4 & 1,1 & 1,4 & 0,4 & 0,3 & 24,1 \\
\hline Mean & 7,3 & 7,6 & 6,9 & 0,2 & 3,1 & Mean & 1,1 & 1,8 & 0,3 & 0,4 & 39,2 \\
\hline N2P1 & 6,9 & 7,3 & 6,6 & 0,2 & 2,9 & N2P1 & 0,8 & 1,4 & 0,3 & 0,3 & 36,7 \\
\hline N2P2 & 7,0 & 7,4 & 6,7 & 0,3 & 3,7 & $\mathrm{~N} 2 \mathrm{P} 2$ & 0,9 & 1,3 & 0,3 & 0,4 & 41,4 \\
\hline N2P3 & 7,3 & 7,6 & 6,4 & 0,4 & 5,2 & N2P3 & 0,8 & 1,6 & 0,2 & 0,5 & 58,8 \\
\hline
\end{tabular}


Table 1 (continued)

\begin{tabular}{|c|c|c|c|c|c|c|c|c|c|c|c|}
\hline \multirow[t]{2}{*}{ Water spring sampling point } & Mean & Maximum & Minimum & Std. Dev. & $\mathrm{CV}$ & Water spring sampling point & Mean & Maximum & Minimum & \multirow[t]{2}{*}{ Std. Dev. } & \multirow[t]{2}{*}{$\mathrm{CV}$} \\
\hline & \multicolumn{6}{|c|}{ Temperature $\left({ }^{\circ} \mathrm{C}\right)$} & \multicolumn{3}{|c|}{ Electrical conductivity $\left(\mu \mathrm{S} \mathrm{cm}^{-1}\right)$} & & \\
\hline N2P4 & 7,4 & 7,6 & 6,9 & 0,2 & 3,1 & $\mathrm{~N} 2 \mathrm{P} 4$ & 0,7 & 1,0 & 0,3 & 0,3 & 38,2 \\
\hline Mean & 7,2 & 7,5 & 6,7 & 0,3 & 3,7 & Mean & 0,8 & 1,3 & 0,3 & 0,3 & 43,8 \\
\hline N3P1 & 5,1 & 6,0 & 4,7 & 0,4 & 6,9 & N3P1 & 2,2 & 3,8 & 1,1 & 0,7 & 33,8 \\
\hline N3P2 & 5,3 & 5,6 & 5,1 & 0,2 & 3,4 & N3P2 & 3,2 & 5,6 & 1,5 & 1,4 & 43,1 \\
\hline N3P3 & 5,6 & 6,1 & 5,1 & 0,3 & 5,2 & N3P3 & 5,5 & 14,9 & 2,5 & 4,1 & 74,9 \\
\hline N3P4 & 5,6 & 6,2 & 5,1 & 0,3 & 5,5 & N3P4 & 2,8 & 6,4 & 1,3 & 1,3 & 47,7 \\
\hline Mean & 5,4 & 6,0 & 5,0 & 0,3 & 5,3 & Mean & 3,4 & 7,7 & 1,6 & 1,9 & 49,9 \\
\hline N4P1 & 6,5 & 7,3 & 5,8 & 0,4 & 5,7 & N4P1 & 3,6 & 6,6 & 2,3 & 1,3 & 37,4 \\
\hline N4P2 & 6,7 & 7,0 & 6,3 & 0,2 & 2,8 & N4P2 & 3,2 & 6,9 & 1,9 & 1,4 & 44,7 \\
\hline N4P3 & 6,7 & 7,2 & 6,2 & 0,3 & 4,6 & N4P3 & 7,5 & 16,0 & 2,3 & 5,3 & 69,8 \\
\hline N4P4 & 6,8 & 7,1 & 6,5 & 0,2 & 3,1 & N4P4 & 5,1 & 11,5 & 2,1 & 3,0 & 58,3 \\
\hline \multirow[t]{2}{*}{ Mean } & 6,7 & 7,2 & 6,2 & 0,3 & 4,1 & Mean & 4,8 & 10,3 & 2,2 & 2,7 & 52,5 \\
\hline & \multicolumn{6}{|c|}{ Nitrite $\left(\mathrm{mg} \mathrm{L}^{-1}\right)$} & \multicolumn{5}{|c|}{ Iron ( $\left.\mathrm{mg} \mathrm{L}^{-1}\right)$} \\
\hline N1P1 & 0,0 & 0,0 & 0,0 & 0,0 & 40,0 & N1P1 & 0,4 & 0,9 & 0,1 & 0,3 & 70,5 \\
\hline N1P2 & 0,0 & 0,0 & 0,0 & 0,0 & 75,0 & N1P2 & 0,3 & 0,5 & 0,1 & 0,2 & 55,2 \\
\hline N1P3 & 0,0 & 0,0 & 0,0 & 0,0 & 40,0 & N1P3 & 0,2 & 0,7 & 0,1 & 0,2 & 104,8 \\
\hline $\mathrm{N} 1 \mathrm{P} 4$ & 0,0 & 0,0 & 0,0 & 0,0 & 66,7 & $\mathrm{~N} 1 \mathrm{P} 4$ & 0,3 & 0,9 & 0,0 & 0,3 & 100,0 \\
\hline Mean & 0,0 & 0,0 & 0,0 & 0,0 & 55,4 & Mean & 0,3 & 0,8 & 0,1 & 0,3 & 82,6 \\
\hline N2P1 & 0,0 & 0,0 & 0,0 & 0,0 & 75,0 & $\mathrm{~N} 2 \mathrm{P} 1$ & 0,5 & 1,0 & 0,2 & 0,3 & 61,7 \\
\hline $\mathrm{N} 2 \mathrm{P} 2$ & 0,0 & 0,0 & 0,0 & 0,0 & 233,3 & $\mathrm{~N} 2 \mathrm{P} 2$ & 0,5 & 0,8 & 0,2 & 0,2 & 47,9 \\
\hline N2P3 & 0,0 & 0,0 & 0,0 & 0,0 & 50,0 & N2P3 & 0,5 & 1,0 & 0,1 & 0,3 & 63,3 \\
\hline $\mathrm{N} 2 \mathrm{P} 4$ & 0,0 & 0,0 & 0,0 & 0,0 & 140,0 & $\mathrm{~N} 2 \mathrm{P} 4$ & 0,5 & 0,9 & 0,1 & 0,3 & 62,2 \\
\hline Mean & 0,0 & 0,0 & 0,0 & 0,0 & 124,6 & Mean & 0,5 & 0,9 & 0,1 & 0,3 & 58,8 \\
\hline N3P1 & 0,0 & 0,0 & 0,0 & 0,0 & 43,8 & N3P1 & 2,7 & 3,0 & 1,1 & 0,7 & 25,4 \\
\hline N3P2 & 0,0 & 0,1 & 0,0 & 0,0 & 63,0 & N3P2 & 2,3 & 3,0 & 0,6 & 0,8 & 35,8 \\
\hline N3P3 & 0,4 & 0,1 & 0,0 & 0,0 & 7,1 & N3P3 & 2,6 & 3,0 & 1,3 & 0,7 & 28,6 \\
\hline N3P4 & 0,0 & 0,0 & 0,0 & 0,0 & 52,4 & N3P4 & 2,5 & 3,0 & 1,5 & 0,6 & 25,1 \\
\hline Mean & 0,1 & 0,1 & 0,0 & 0,0 & 41,6 & Mean & 2,5 & 3,0 & 1,1 & 0,7 & 28,7 \\
\hline N4P1 & 0,0 & 0,0 & 0,0 & 0,0 & 41,7 & N4P1 & 2,4 & 3,0 & 1,1 & 0,8 & 31,3 \\
\hline N4P2 & 0,0 & 0,1 & 0,0 & 0,0 & 65,2 & N4P2 & 2,4 & 3,0 & 0,8 & 0,8 & 34,6 \\
\hline N4P3 & 0,0 & 0,1 & 0,0 & 0,0 & 84,2 & N4P3 & 2,7 & 3,0 & 1,7 & 0,5 & 20,0 \\
\hline N4P4 & 0,0 & 0,1 & 0,0 & 0,0 & 60,0 & N4P4 & 2,8 & 3,0 & 2,1 & 0,4 & 12,4 \\
\hline Mean & 0,0 & 0,1 & 0,0 & 0,0 & 62,8 & Mean & 2,6 & 3,0 & 1,4 & 0,6 & 24,6 \\
\hline
\end{tabular}

Note: $\mathrm{N}$ refers to the water spring number, while $\mathrm{P}$ to the water sampling point; $\mathrm{CV}=$ coefficient of variation (std. dev. / mean, [\%]).

climate within the basin (Singh et al., 2004). Tropical streams differ ecologically from temperate ones, since streams in the tropics typically receive higher solar radiation and more intense rainfall, with warmer water and often relatively predictable floods; moreover, they show a higher biodiversity than their temperate equivalents (Dudgeon, 1999), which suggested to investigate the water quality of tropical streams as affected by natural and human-induced stresses.

In general, from the noticeable variations of the physico-chemical parameters of water quality related to the effects of land use, it is evident that water courses are very sensitive to changes in the environment and this is particularly true in the portions of the stream where vegetation is removed or increasingly modified. Also this study revealed

Table 2

Comparison between linear mixed models (LMM) applied to water quality parameters of water springs in Pimenta creek (São Paulo State, Brazil).

\begin{tabular}{llll}
\hline \multirow{2}{*}{ Water quality parameters } & \multicolumn{1}{l}{$\operatorname{Pr}\left(>\chi^{2}\right)$} & \\
\cline { 2 - 4 } & $\mathrm{LMM}_{\mathrm{s} \cdot \mathrm{d}} \mathrm{Vs} \mathrm{LMM}_{\mathrm{s}}$ & $\mathrm{LMM}_{\mathrm{s} \cdot \mathrm{d}} \mathrm{Vs} \mathrm{LMM}_{\mathrm{d}}$ & $\mathrm{LMM}_{\mathrm{s}} \mathrm{Vs} \mathrm{LMM}_{\mathrm{d}}$ \\
\hline Temperature & 0.94 & $<0.001^{* * *}$ & 1 \\
Electrical conductivity & $<0.001^{* * * *}$ & $<0.001^{* * *}$ & 1 \\
Total Dissolved Solids & $<0.001^{* * *}$ & $<0.001^{* * *}$ & 1 \\
Salinity & $<0.001^{* * *}$ & $<0.001^{* * *}$ & 1 \\
Turbidity & $<0.001^{* * * *}$ & $<0.001^{* * *}$ & 1 \\
Color & $<0.001^{* * * *}$ & $<0.001^{* * *}$ & 1 \\
pH & $<0.001^{* * *}$ & $<0.001^{* * *}$ & 1 \\
Nitrate & $<0.001^{* * * *}$ & $<0.001^{* * *}$ & 1 \\
Nitrite & $0.01^{*}$ & $<0.001^{* * *}$ & 1 \\
Iron & 0.34 & $<0.001^{* * *}$ & 1 \\
\hline
\end{tabular}

Notes: in bold characters the significant differences are highlighted; ${ }^{* * *},{ }^{* * *}$ significant difference at $\mathrm{p}<0.05,0.01$ and 0.001 , respectively; $\mathrm{LMM}_{\mathrm{s} \cdot \mathrm{d}}=$ linear mixed model applied to both water spring and sampling point; $\mathrm{LMM}_{\mathrm{s}}=$ linear mixed model applied to water spring; $\mathrm{LMM}_{\mathrm{d}}=$ linear mixed model applied to sampling point. a significant incidence of the different land uses and conservation levels of riparian vegetation as well as the distance of sampling points on water quality, as indicated by the significant differences detected in the majority of the analysed parameters.

First of all, the implementation and reciprocal comparison of three different LMMs showed that, when water quality is monitored in tropical streams, not only the land use of the spring surroundings, but also the sampling distance can play an influence. As a matter of fact, if $\mathrm{LMM}_{\mathrm{s}}$ take into account as variability factor only sampling point or land use effects, the difference in water quality parameters are always not significant (see comparison of $\mathrm{LMM}_{\mathrm{s}}$ and $\mathrm{LMM}_{\mathrm{d}}$ models in Table 2). Viceversa, a 2-level LMM highlights correctly the variability of water quality which depend on both land use and distance from water source (see $\mathrm{LMM}_{\mathrm{s} \cdot \mathrm{d}}$ in Table 2).

As regards the effects of land use on water quality parameters, the study has demonstrated that water temperature and iron concentration were not significantly variable among forest (spring "N1"), disturbed vegetation ("N2"), pasture ("N3") and agricultural land uses ("N4"); however, the water springs with riparian vegetation showed slightly low temperature throughout the year (even though not significantly) because of the shadowing of forest canopies. The spring "N4", unprotected from vegetation, and "N3", with small vegetation, were exposed to direct solar radiation and consequently the temperatures rise up (Arcova and Cicco, 1999).

In comparison to the international standards (e.g. those of USEPA), the water quality of the analysed spring was generally good with the exception of iron concentration. This element, an important indicator of geogenic conditions, becomes soluble by redox processes in soils and sediment (Vuori, 1995) and, thus, the stream water concentration tends to depend on the particular hydrological situation rather than on land use (Kändler et al., 2017). The iron concentration of Pimenta 
Table 3

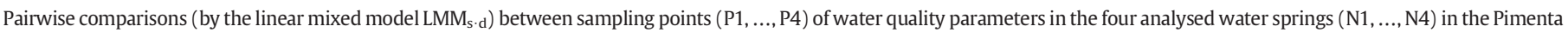
creek (São Paulo State, Brazil).

\begin{tabular}{|c|c|c|c|c|c|c|c|c|c|c|c|}
\hline \multicolumn{2}{|c|}{$\begin{array}{l}\text { Water } \\
\text { spring/sampling point }\end{array}$} & \multicolumn{2}{|c|}{ Temperature } & \multicolumn{2}{|c|}{$\begin{array}{l}\text { Electrical } \\
\text { conductivity }\end{array}$} & \multicolumn{2}{|c|}{ Total dissolved solids } & \multicolumn{2}{|l|}{ Salinity } & \multicolumn{2}{|c|}{ Turbidity } \\
\hline & & $\mathrm{z}$ value & $\operatorname{Pr}(>|z|)$ & $\mathrm{z}$ value & $\operatorname{Pr}(>|z|)$ & $\mathrm{z}$ value & $\operatorname{Pr}(>|z|)$ & $\mathrm{z}$ value & $\operatorname{Pr}(>|z|)$ & $\mathrm{z}$ value & $\operatorname{Pr}(>|z|)$ \\
\hline N1P1 & N1P2 & 0.2 & 1 & -1.7 & 0.9 & -2.08 & 0.77 & -1.38 & 0.99 & 0.41 & 1 \\
\hline N1P1 & N1P3 & 0.3 & 1 & -1.14 & 0.9 & -1.45 & 0.98 & -0.79 & 1 & 0.35 & 1 \\
\hline N1P1 & N1P4 & 0.22 & 1 & -0.5 & 1 & -0.46 & 1 & -0.05 & 1 & 0.05 & 1 \\
\hline N1P2 & N1P3 & 0.09 & 1 & 0.55 & 1 & 0.59 & 1 & -23.68 & 1 & 0.76 & 1 \\
\hline N1P2 & N1P4 & 0.02 & 1 & 1.2 & 0.9 & 1.62 & 0.97 & -25.18 & 0.99 & -0.46 & 1 \\
\hline N1P3 & N1P4 & -0.08 & 1 & 0.64 & 1 & 1.03 & 0.99 & 0.75 & 1 & 0.3 & 1 \\
\hline N2P1 & N2P2 & -0.31 & 1 & -1.85 & 0.9 & -1.99 & 0.83 & -1.44 & 0.99 & 1.09 & 0.99 \\
\hline N2P1 & N2P3 & -0.61 & 1 & -2.05 & 0.8 & -2.25 & 0.66 & 1.8 & 0.99 & 0.99 & 1 \\
\hline N2P1 & $\mathrm{N} 2 \mathrm{P} 4$ & -0.96 & 0.9 & -1.59 & 0.9 & -1.86 & 0.89 & -1.36 & 0.92 & 0.87 & 1 \\
\hline N2P2 & N2P3 & -0.3 & 1 & -0.2 & 1 & -0.25 & 1 & 0.35 & 1 & -0.09 & 1 \\
\hline N2P2 & N2P4 & -0.65 & 1 & 0.26 & 1 & 0.14 & 1 & 0.08 & 1 & -0.21 & 1 \\
\hline N2P3 & N2P4 & -0.36 & 1 & 0.46 & 1 & 0.38 & 1 & 0.44 & 1 & -0.12 & 1 \\
\hline N3P1 & N3P2 & 0.89 & 1 & -2.64 & 0.4 & -2.81 & $<0.01^{* * *}$ & -2.08 & 0.78 & 7.93 & $<0.01^{* * *}$ \\
\hline N3P1 & N3P3 & 0.65 & 1 & -3.56 & $0.03^{*}$ & -4.01 & $<0.01^{* *}$ & -2.86 & 0.23 & 73.31 & $<0.01^{* * *}$ \\
\hline N3P1 & N3P4 & 0.3 & 1 & -3.06 & 0.1 & 3.84 & $0.01^{*}$ & -2.95 & 0.19 & 7.21 & $<0.01^{* * *}$ \\
\hline N3P2 & N3P3 & -0.24 & 1 & -0.92 & 0.9 & 1.27 & 0.99 & -0.79 & 1 & -0.62 & 1 \\
\hline N3P2 & N3P4 & -0.6 & 1 & -0.42 & 1 & -1.03 & 0.99 & -0.87 & 1 & -0.71 & 1 \\
\hline N3P3 & N3P4 & 0.36 & 1 & 0.5 & 1 & 0.24 & 1 & -0.84 & 1 & -0.1 & 1 \\
\hline N4P1 & N4P2 & -1.09 & 0.9 & -3.43 & $0.05^{*}$ & -2.37 & 0.57 & -3.37 & 0.06 & 0.58 & 1 \\
\hline N4P1 & N4P3 & -0.88 & 1 & -2.95 & 0.2 & -4 & $<0.01^{* *}$ & -4.44 & $<0.01^{* * *}$ & 1.5 & 0.98 \\
\hline N4P1 & N4P4 & -1.85 & 0.9 & -4.74 & $<0.01^{*}$ & -3.9 & $<0.01^{* *}$ & -4.97 & $<0.01^{* * *}$ & 0.9 & 1 \\
\hline N4P2 & N4P3 & 0.2 & 1 & 0.48 & 1 & -1.63 & 0.96 & -1.07 & 0.99 & 0.92 & 1 \\
\hline N4P2 & N4P4 & -0.76 & 1 & -1.31 & 0.9 & -1.53 & 0.98 & -1.6 & 0.97 & 0.32 & 1 \\
\hline N4P3 & N4P4 & -0.96 & 0.9 & -1.79 & 0.92 & 0.1 & 1 & -0.53 & 1 & -0.6 & 1 \\
\hline \multirow{2}{*}{\multicolumn{2}{|c|}{$\begin{array}{l}\text { Water } \\
\text { spring/sampling } \\
\text { point }\end{array}$}} & \multicolumn{2}{|l|}{ Color } & \multicolumn{2}{|l|}{$\mathrm{pH}$} & \multicolumn{2}{|l|}{ Nitrate } & \multicolumn{2}{|l|}{ Nitrite } & \multicolumn{2}{|l|}{ Iron } \\
\hline & & z value & $\operatorname{Pr}(>|z|)$ & z value & $\operatorname{Pr}(>|z|)$ & \multicolumn{2}{|l|}{ z value } & z value & $\operatorname{Pr}(>|z|)$ & z value & $\operatorname{Pr}(>|z|)$ \\
\hline N1P1 & N1P2 & 2.16 & 0.73 & 1.3 & 0.99 & -0.55 & 1 & -0.56 & 1 & -1.28 & 0.99 \\
\hline N1P1 & N1P3 & 1.87 & 0.89 & 5.57 & $<0.01^{* * *}$ & -0.57 & 1 & 0.39 & 1 & -2.49 & 0.48 \\
\hline N1P1 & N1P4 & 2.15 & 0.73 & 5.26 & $<0.01^{* * *}$ & -0.36 & 1 & 0.47 & 1 & -1.69 & 0.95 \\
\hline N1P2 & N1P3 & 0.29 & 1 & 4.27 & $<0.01^{* * *}$ & -0.01 & 1 & 0.97 & 0.99 & -1.29 & 0.99 \\
\hline N1P2 & N1P4 & -0.01 & 1 & 3.96 & $<0.01^{* * *}$ & 0.19 & 1 & 1.05 & 0.99 & -0.41 & 1 \\
\hline N1P3 & N1P4 & 0.28 & 1 & -0.31 & 1 & 0.21 & 1 & 0.09 & 1 & 0.8 & 1 \\
\hline N2P1 & N2P2 & 0.7 & 1 & 1.27 & 0.99 & 0.21 & 1 & -0.82 & 1 & 0.22 & 1 \\
\hline N2P1 & $\mathrm{N} 2 \mathrm{P} 3$ & 1.2 & 0.99 & 4.36 & $<0.01^{* * *}$ & -0.12 & 1 & 0.8 & 1 & 0.05 & 1 \\
\hline N2P1 & N2P4 & 2.19 & 0.71 & 5.25 & $<0.01^{* * *}$ & -0.4 & 1 & 0.69 & 1 & -0.22 & 1 \\
\hline N2P2 & N2P3 & 0.5 & 1 & 3.01 & 0.13 & -0.33 & 1 & 1.63 & 0.96 & -0.16 & 1 \\
\hline N2P2 & $\mathrm{N} 2 \mathrm{P} 4$ & 1.49 & 0.98 & 3.98 & $<0.01^{* * *}$ & -0.61 & 1 & 1.51 & 0.98 & -0.43 & 1 \\
\hline N2P3 & $\mathrm{N} 2 \mathrm{P} 4$ & 0.99 & 1 & 0.89 & 1 & -0.29 & 1 & -0.12 & 1 & -0.27 & 1 \\
\hline N3P1 & N3P2 & 5.16 & $<0.01^{* * *}$ & 3.03 & 0.16 & 1.77 & 0.93 & 2.16 & 0.73 & -1.54 & 0.98 \\
\hline N3P1 & N3P3 & 6 & $<0.01^{* * *}$ & 5.97 & $<0.01^{* * *}$ & 4.72 & $<0.01^{* * *}$ & 3.93 & $<0.01^{* *}$ & -0.35 & 1 \\
\hline N3P1 & N3P4 & 5.81 & $<0.01^{* * *}$ & 6.38 & $<0.01^{* * *}$ & 1.04 & 0.99 & 1.11 & 0.99 & -0.58 & 1 \\
\hline N3P2 & N3P3 & 0.84 & 1 & 2.95 & 0.19 & 2.95 & 0.19 & 1.77 & 0.92 & 1.19 & 0.99 \\
\hline N3P2 & N3P4 & 0.65 & 1 & 3.35 & 0.06 & -0.73 & 1 & -1.04 & 0.99 & 0.97 & 1 \\
\hline N3P3 & N3P4 & -0.19 & 1 & 0.4 & 1 & -3.68 & $0.02^{*}$ & -2.82 & 0.26 & -0.23 & 1 \\
\hline N4P1 & N4P2 & -0.21 & 1 & 2.29 & 0.63 & -0.68 & 1 & -0.31 & 1 & -0.11 & 1 \\
\hline N4P1 & N4P3 & 0.27 & 1 & 2.42 & 0.53 & 4.57 & $<0.01^{*}$ & 1.92 & 0.87 & 0.98 & 1 \\
\hline N4P1 & N4P4 & 0.71 & 1 & 3.62 & $0.02 *$ & 1.97 & 0.84 & 0.85 & 1 & 1.74 & 0.94 \\
\hline N4P2 & N4P3 & 0.48 & 1 & 0.13 & 1 & 5.24 & $<0.01^{* * *}$ & 2.23 & 0.68 & 1.09 & 0.99 \\
\hline N4P2 & N4P4 & 0.92 & 1 & 1.33 & 0.99 & 2.64 & 0.37 & 1.16 & 0.99 & 1.85 & 0.9 \\
\hline N4P3 & N4P4 & 0.44 & 1 & 1.2 & 0.99 & -2.6 & 0.4 & -1.07 & 0.99 & 0.76 & 1 \\
\hline
\end{tabular}

Notes: in bold characters the significant differences are highlighted; *, ${ }^{* *}{ }^{* * *}$ significant difference at $\mathrm{p}<0.05,0.01$ and 0.001 , respectively.

creek showed some variations among the springs, even though not significantly: water quality of spring "N1" was similar as "N2" and these latter springs differed from "N3" and "N4". The values of iron concentration measured at the springs "N1" and "N2" highlighted that the presence of the riparian vegetation, preserved or even disturbed, influenced this parameter of spring water; on the contrary, the degraded vegetation of spring "N4" and bamboo with a small strip of degraded vegetation in "N3" showed that the absence or low strips of riparian complexes in a degraded state resulted in an increase of iron values, able to overcome the acceptance limits (suggested by USEPA standards) for this water quality parameter.

Compared to water springs with riparian vegetation (preserved, "N1", or degraded, "N2"), turbidity, color, pH and nitrate concentrations were much higher (that is, in "N3" and "N4"). The water pH was close to neutrality in the springs with riparian vegetation (accordingly to Donadio et al., 2005, who found pH close to 7 in water springs with tropical natural vegetation), showing a more preserved aquatic environment, while water was quite acid (due to organic acid pouring with wastewater of indigenous origin) in the other springs, which indicated possible water pollution. At the downstream sampling points, an increase of the concentration of color, turbidity and suspended solids was observed related with agricultural ditches triggering the coupling of agricultural hillslopes and stream (Slattery et al., 2002). In tropical forest environments, also Primavesi et al. (2002) and Donadio et al. (2005) reported higher values of turbidity in microbasins with agricultural land use than in forested areas, thus evidencing the function of the 

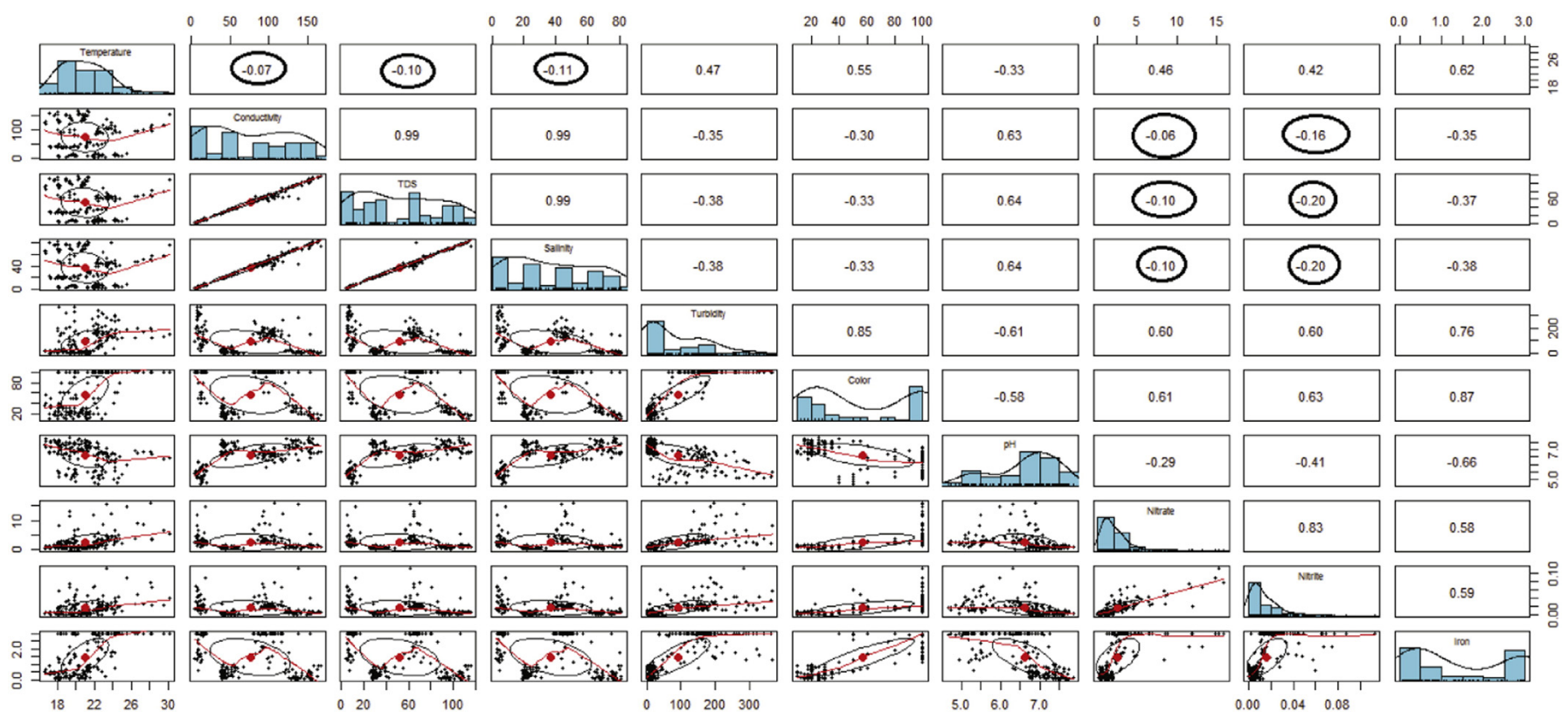

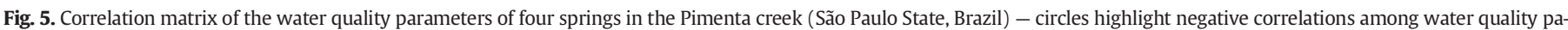

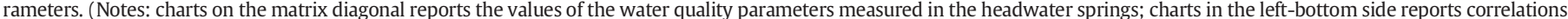

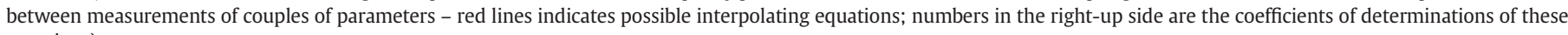
equations).

riparian forest in reducing solids supply from sources to stream water. The increase in turbidity values due to the scarcity of riparian forest were also observed in the study of Arcova and Cicco (1999) in a tropical and agricultural microbasin, which showed also higher color values and suspended sediments in water of the stream interfering with the presence of a road (as in spring "N4" of our study). Also Donadio et al. (2005) found lower values of the water color in tropical streams of riparian forest compared to other land uses. Many Authors reported that farmland is responsible for water pollution, while, on the contrary, forested areas show negative correlations with most ions (e.g. Bahar et al., 2008; Zhou et al., 2012; Xia et al., 2012; Kändler et al., 2017). As Keesstra et al. (2012) found, the riparian zone has a significant effect on water and sediment transport in headwater catchments, since high roughness in natural rivers due to vegetation and geomorphological attributes may generate drag on flowing water. This is also in accordance with Gao (2008), who showed that the riparian vegetation in headwater catchments play an important role in the resulting water and sediment dynamics of rivers further downstream; in general, vegetation of riparian zones has a demonstrated buffer capacity for avoiding the transfer of diffuse contaminants to surface waters (Connolly et al., 2015). Since headwater streams are particularly closely coupled with adjacent riparian and terrestrial environments, because of the higher ratio of aquatic-riparian interface and the sensitivity of riparian zones towards river basin ecohydrology (Bombino et al., 2014; Wohl, 2017), riparian buffer strips and their structure are critical for maintaining water functions and minimising eutrophication (Boëchat et al., 2013; Parron et al., 2010; Fernandes et al., 2014; Hunke et al., 2015b). Thus, under the catchment management point of view, riparian vegetation should be promoted in stream channels and intensive agricultural uses in adjacent areas should be avoided, in order to not alter water quality (Rodrigues et al., 2018).

Differences in Electrical conductivity, Total Dissolved Solids and salinity (all of them strongly correlated each other, as highlighted above) among the different land uses close to the four water springs do not seem to be in relation to human-induced changes (affecting "N3" and "N4") or lower disturbance in headwaters (as in "N1" and "N2"); as a matter of fact, the highest values were surveyed in the preserved spring, while the lowest parameters were measured in the partially degraded headwaters. The determinant factor for the electrical conductivity values may be the geology of the sites, that, for instance, is constituted by rocks resistant to weathering, such as granites and gneisses, in "N1" (highest electrical conductivity), and soils in advanced stage of weathering in "N2" (lowest EC) (Arcova and Cicco, 1999). Extremely weathered, undisturbed watersheds are characterized by very low in-stream ionic concentrations (and therefore electrical conductivity) often dominated by the Calcium ion (Markewitz et al., 2006).

Nutrients such as inorganic nitrogen (ammonia, nitrate and nitrite) are important factors affecting water quality, since they play, together the bioavailable forms of phosphorous, an important role in the eutrophication process in surface waters (Soulsby et al., 2001; Şener et al., 2017). The higher concentrations of nitrites and mainly of nitrates in springs "N3" (pastured) and "N4" (cropped) - even though low compared to USEPA water quality standards - can be explained, as for $\mathrm{pH}$, by the fact that the sites were heterogeneous in land use and these parameters are sensitive to denudation of riparian vegetation and nitrogen-based fertiliser use, since fertilizers and animal manure (rich in nitrogen) is usually transported downstream by surface runoff to the waterways. The spring "N3" had a domestic sewage at its headwater; this fact may have contributed to the spatial variation of turbidity and color, increasing the presence of suspended solids in water and the sediment transport downstream, which interfere with light penetration through the water. Effluents pouring into streams is in fact an important factor that controls water quality parameters (Castro and Mendonça, 2004). Many different studies have shown that agricultural land uses at catchment scale is a primary predictor for water quality compounds (Smart et al., 1998; Ferrier et al., 2001; Ahearn et al., 2005). An increase in electrical conductivity and inorganic $\mathrm{N}$-forms in surface water was detected in most reviewed studies due to anthropogenic inputs from fertilisation and liming. Silva et al. (2011) detected higher nitrite concentrations and water conductivity in tropical rural streams compared to natural low order catchments and concluded that agricultural land use had a measurable impact on solute loads in the river system (Hunke et al., 2015b). These latter Authors from their water sampling results demonstrated the significant impact of agricultural use on water quality, especially for nitrate and nitrite concentrations. For small first-order pasture catchments, Gücker et al. (2009) 
found significantly higher electrical conductivities and $\mathrm{NO}_{3}$ compared with natural streams. The concentrations of $\mathrm{NO}_{3}$ and $\mathrm{NO}_{2}$ in rural streams were as much as 1.5 times higher, and they differed significantly from natural streams (Hunke et al., 2015a). Fonseca et al. (2013) presented similar findings in low-order pristine streams.

Conversely, an improvement in the water quality is usually observed in relation to the amount of nitrate and nitrite in the microcatchments with dense vegetation cover and in an advanced regeneration phase. $\mathrm{Ni}-$ trate concentrations in surface water of Brazilian forests (e.g. in Cerrado) were orders of magnitude lower compared with concentrations measured in European rivers under land use change (Hunke et al., 2015a). In a stream with gallery forest within an ecological reserve near Brasilía, Parron et al. (2010) found only very low N concentrations, whereas nitrates were not detectable, due to the fact that forest has a high filtering capacity. Moreover, a dilution effect of $\mathrm{NaNO}_{2}, \mathrm{NO}_{2}$ and $\mathrm{NO}_{2}$ - $\mathrm{N}$ as well as Total Dissolved Solids (and, as a consequence, of electrical conductivity and salinity) was observed downstream of tropical rivers (Rodrigues et al., 2018). Analyzing the impacts of human activities in reforested basins, Castro and Mendonça (2004) found an improvement in the quality of in relation to the amount of nitrate and nitrite in microbasins with dense vegetation cover and in advanced regeneration phase. The same Authors also recorded higher amounts of nitrate due to agricultural practices and soil exposure by low effective protection coverage, in addition to that of fertilizers.

As different studies have demonstrated, land use changes (i.e. from forest to agricultural land uses) may alter and increase water and sediment connectivity, thus changing water quality along streams (Parsons et al., 2015; Masselink et al., 2017a; Masselink et al., 2017b). From the study it was evident that most of the water quality parameters in agriculture-dominated sites had higher concentrations than those in forest-dominated sites, as also stated by Ding et al. (2015). Therefore, the land use type had a significant weight not only in the correlation coefficients for each water quality characteristic but also in the degree of influence of land use itself on each water quality variable (Yu et al., 2016). However, some important caveats apply to studies of the relationship between land use and stream water quality: when conducting a Pearson's correlation analysis on this type of relationship, the conclusion that the land use type was the primary driver of stream water quality must be made with caution (Yu et al., 2016). In addition, forecasting changes in stream water quality in response to changes in land use type may run the risk that the relationship would alter over time owing to changes in some specific practices or the environment itself (Allen, 2004).

With reference to the water quality variability as function of the sampling distance from the spring, the spatial variations were significant for the majority of the couples of points in relation to $\mathrm{pH}$, demonstrating the heterogeneity of the site and the sensitivity of this parameter, but not for temperature and iron concentrations. Water temperature and iron concentrations remained unaltered within each spring (that is, within the same land use) regardless of the distance of sample collection. Nitrate concentration as well as Electrical conductivity, Total Dissolved Solids and salinity had significant spatial variations only in the two water springs considered to be degraded ("N3" and "N4"), as direct consequence of both human-induced changes (pasture, agriculture and wastewater pouring) and lower presence of riparian vegetation. Water color and turbidity significantly differed according to the distance only in pastured spring. The noticeable relation between flow quality and the effect of the distance was detected also by Castro and Mendonça (2004) and Rodrigues et al. (2018), who found an important relationship between discharge and the distance effect on water quality parameters: the study of these latter Authors indicated that the measured water quality parameters varied among the different sampling points from the headwater sampling point $(0 \mathrm{~m})$ to the last downstream sampling point $(2500 \mathrm{~m})$.

Overall, the study showed that, for a preserved water spring covered by riparian forest, more or less preserved (as the spring "N1" and "N2" are), only pH of water may suffer from some alterations between the surroundings of spring (points "P1" and "P2") and other zones at a distance from the source ("P3" and "P4"), being the differences among the other water quality parameters practically not appreciable. Conversely, the significant variations of parameters detected for the agricultural spring (e.g. turbidity, color, total suspended solids) demonstrate the increase of instability of water quality parameters with distance in a spring degraded from its headwater. Finally, in the last spring (degraded as the previous one) the lower differences (mainly in electrical conductivity, Total Dissolved Solids and salinity) in the surveyed paramters evidenced less noticeable spatial variations of water quality in pasture.

Our results are in tune with those of studies carried out in temperate systems, which have demonstrated that riparian forest buffers act on water quality, by filtering sediment and nutrients from agricultural runoff and providing shade that moderates stream temperatures and regulates instream primary production (Karr and Schlosser, 1978; Peterjohn and Correll, 1984; Osborne and Kovacic, 1993). However, although riparian forest buffers are expected to provide similar functions in tropical systems, studies documenting relationships between forest buffers and river ecosystem components in the tropics are conspicuously lacking (Lorion and Kennedy, 2009); our study tried to fill this gap, suggesting how, in tropical headwaters of Mata Atlantica (one of the most threatened biome in Brazil, SOS Mata Atlantica and INPE, 2013), forested riparian zones could significantly reduce the impacts of deforestation - by agricultural and pasture activities - on tropical streams (Pringle and Scatena, 1999; Benstead et al., 2003).

\section{Conclusions}

In spite of a generally good water quality (except for the iron concentration), the monitoring of water quality in a small headwater of the tropical environment showed a large variability of many parameters among the different land uses and sampling points. Both these factors play an important role in explaining water quality variability, as showed by the comparison among three linear mixed models. Among the analysed parameters, some of which strongly correlated each other (mainly electrical conductivity, Total Dissolved Solids and salinity, but also color, turbidity and iron concentrations), $\mathrm{pH}$ was found to be the water quality parameter with the highest spatial variability among sampling points. The other parameters evaluated showed variations mainly due to the effects of the different land uses, but also to the distance from water spring. In general, the study demonstrated the instability of the water quality parameters in spring degraded from its headwater. The water springs with developed riparian vegetation of natural forest (in a preserved or even disturbed conservation level) showed the best conditions in the aquatic environment (lower temperature, turbidity, color, nitrite and nitrate concentrations, neutral $\mathrm{pH}$ ). Conversely, compared to vegetated surroundings, in the water springs with pasture or agricultural activities a general worsening of water quality was detected (worse turbidity, color, $\mathrm{pH}$, nitrite and nitrate concentrations).

On the whole, the study has demonstrated how much aquatic environment is sensitive to changes in the environment, confirming findings of literature. It has also been highlighted the importance of riparian vegetation effects for conservation of water quality of tropical headwater catchments, where, instead, agriculture and pasture may represent a threat against natural resource preservation. The study can serve as a monitoring model in compared to other impacted watersheds (Arcova and Cicco, 1999) and the values of the water quality parameters achieved may represent a reliable database to support the development of conservation and management strategies for tropical headwaters. However, it should be noted that the use of water as a qualitative indicator requires further studies to verify that the other factors that may interfere with its quality.

Finally, the monitoring activities of water quality allow us to know and interpret the actual influences played by factors of change (such as land use, spatial and temporal changes) on water quality and riparian 
ecology. Understanding the relationships between water quality and their variability and land use as is necessary to diagnose information on the health of water springs and headwater streams and to support the adoption of the best management strategy (Lessels and Bishop, 2013).

\section{Acknowledgments}

To the CAPES Fundation (Ministério Da Educação CAPES, Brazil) for the study grants; to the employees and trainees of the Experimental Farm of São Manuel (São Paulo State University, Faculty of Agronomic Sciences), the researcher Dr. Valdir de Cicco (Instituto Federal de Educação, Ciência e Tecnologia do Triângulo Mineiro, São Paulo State) and professors Dr. Renato Farias do Valle Junior (Instituto Federal de Educação, Ciência e Tecnologia do Triângulo Mineiro, São Paulo State) and Dr. Raimundo Leite Cruz (São Paulo State University, Faculty of Agronomic Sciences).

\section{References}

Ahearn, D.S., Sheibley, R.S., Dahlgren, R.A., Anderson, M., Jonson, J., Tate, K.W., 2005. Land use and land cover influence on water quality in the last free-flowing river draining the western Sierra Nevada, California. J. Hydrol. 313, 234-247.

Alexander, R.B., Boyer, E.W., Smith, R.A., Schwarz, G.E., Moore, R.B., 2007. The role of headwater streams in downstream water quality. J. Am. Water Resour. Assoc. 43 (1), 41-59.

Allen, J.D., 2004. The influence of land use on stream ecosystems. Annu. Rev. Ecol. Evol. Syst. 35, 257-284.

Amiri, B.J., Nakane, K., 2009. Modeling the linkage between river water quality and landscape metrics for water quality in the Chugoku district of Japan. Water Resour. Manag. 23, 931-956.

Arcova, F.C.S., Cicco, V., 1999. Qualidade da água de microbacia com diferentes usos do solo na região de Cunha, Estado de São Paulo. Scientia Forestalis 56, 125-134.

SOS Atlantic Forest and INPE, 2013. The National Institute for Space Research - Atlas of Forest Remnants of Atlantic Rainforest. Technical Report, São Paulo, Brazil.

Bahar, M.M., Ohmori, H., Yamamuro, M., 2008. Relationship between river water quality and land use in a small river basin running through the urbanizing area of Central Japan. Limnology 9 (1), 19-26.

Baker, A., 2003. Land use and water quality. Hydrol. Process. 17 (12), 2499-2501.

Benstead, J.P., Douglas, M.M., Pringle, C.M., 2003. Relationships of stream invertebrate communities to deforestation in eastern Madagascar. Ecol. Appl. 13, 1473-1490.

Bere, T., Tundisi, J.G., 2011. Influence of land-use patterns on benthic diatom communities and water quality in the tropical Monjolinho hydrological basin, São Carlos-SP, Brazil. Water SA 37 (1), 93-102.

Boëchat, I.G., Paiva, A.B.D.M.D., Hille, S., Gücker, B., 2013. Land-use effects on river habitat quality and sediment granulometry along a 4 th-order tropical river. Rev. Ambiente Água 8 (3), 54-64.

Bombino, G., Gurnell, A.M., Tamburino, V., Zema, D.A., Zimbone, S.M., 2007. A method for assessing channelization effects on riparian vegetation in a Mediterranean environment. River Res. Appl. 23 (6):613-630. https://doi.org/10.1002/rra.1004.

Bombino, G., Boix-Fayos, C., Gurnell, A.M., Tamburino, V., Zema, D.A., Zimbone, S.M., 2014. Check dam influence on vegetation species diversity in mountain torrents of the Mediterranean environment. Ecohydrology 7 (2):678-691. https://doi.org/10.1002/eco.1389.

Bu, H., Meng, W., Zhang, Y., Wan, J., 2014. Relationships between land use patterns and water quality in the Taizi River basin, China. Ecol. Indic. 41, 187-197.

Castro, C.B., Mendonça, A.S.F., 2004. Impactos de ações antrópicas em bacias de mananciais rurais de montanha sobre parâmetros de qualidade de água. Rev. Brasil. de Recurs. Hídric. 9 (1), 17-26.

Connolly, N.M., Pearson, R.G., Loong, D., Maughan, M., Brodie, J., 2015. Water quality variation along streams with similar agricultural development but contrasting riparian vegetation. Agric. Ecosyst. Environ. 213, 11-20.

Cunha, A.R., Martins, D., 2009. Classificação climática para os municípios de Botucatu e São Manuel, SP. Irriga 14 (1), 1-11.

Ding, J., Jiang, Y., Fu, L., Liu, Q., Peng, Q., Kang, M., 2015. Impacts of land use on surface water quality in a subtropical River Basin: a case study of the Dongjiang River Basin, Southeastern China. Water 7 (8), 4427-4445.

Donadio, N.M.M., Galbiatti, J.A., Paula, R.C., 2005. Qualidade da água de nascentes com diferentes usos do solo na bacia hidrográfica do córrego Rico, São Paulo, Brasil. Engenharia Agrícola 25 (1), 115-125.

Downing, J.A., Cole, J.J., Duarte, C.M., Middelburg, J.J., Melack, J.M., Prairie, Y.T., Kortelainen, P., Striegl, R.G., McDowell, W.H., Tranvik, L.J., 2012. Global abundance and size distribution of streams and rivers. Inland Waters 2 (4), 229-236.

Dudgeon, D., 1999. Tropical Asian Streams: Zoobenthos, Ecology and Conservation (Vol. 1). University Press, Hong Kong.

Emmett, B.A., Hudson, J.A., Cowar, D.P.A., Reynolds, B., 1994. The impact of a riparian wetland on streamwater quality in a recently afforested upland catchment. J. Hydrol. 162 (3-4), 337-353.

Fernandes, J.D.F., Souza, A.L., Tanaka, M.O., 2014. Can the structure of a riparian forest remnant influence stream water quality? A tropical case study. Hydrobiologia 724 (1), 175-185.
Ferrier, R.C., Edwards, A.C., Hirst, D., Littlewood, I.G., Watts, C.D., Morris, R., 2001. Water quality of Scottish rivers: spatial and temporal trends. Sci. Total Environ. 265, 327-342.

Fonseca, B.M., Mendonça-Galvão, L., Padovesi-Fonseca, C., Abreu, L.M., Fernandes, A.C.M. 2013. Nutrient baselines of Cerrado low-order streams: comparing natural and impacted sites in Central Brazil. Environ. Monit. Assess. 186 (1), 19-33.

Fonseca, B.M., de Mendonça-Galvão, L., 2014. Pristine aquatic systems in a long term ecological research (LTER) site of the Brazilian Cerrado. Environ. Monit. Assess. 186 (12), 8683-8695.

Fujieda, M., Kudoha, T., Cicco, V., Calvarcho, J.L., 1997. Hydrological processes at two subtropical forest catchments: the Serra do Mar, São Paulo, Brazil. J. Hydrol. 196, 26-46.

Gao, P., 2008. Understanding watershed suspended sediment transport. Prog. Phys. Geogr. 32, 243-263.

Gomi, T., Sidle, R.C., Richardson, J.S., 2002. Understanding processes and downstream linkages of headwater systems. Bioscience 52 (10), 905-916.

Gücker, B., Boëchat, I.G., Giani, A., 2009. Impacts of agricultural land use on ecosystem structure and whole-stream metabolism of tropical Cerrado streams. Freshw. Biol. 54, 2069-2085.

Hartemink, A.E., Veldkamp, A., Bai, Z., 2008. Land cover change and soil fertility decline in tropical regions. Turk. J. Agric. For. 32, 195-213.

Hunke, P., Mueller, E.N., Schröder, B., Zeilhofer, P., 2015a. The Brazilian Cerrado: assessment of water and soil degradation in catchments under intensive agricultural use. Ecohydrology 8 (6), 1154-1180.

Hunke, P., Roller, R., Zeilhofer, P., Schröder, B., Mueller, E.N., 2015b. Soil changes under different land-uses in the Cerrado of Mato Grosso, Brazil. Geoderma Reg. 4, 31-43.

Hurley, T., Mazumder, A., 2013. Spatial scale of land-use impacts on riverine drinking source water quality. Water Resour. Res. 49, 1591-1601.

Johnson, L.B., Gage, S.H., 1997. Landscape approaches to the analysis of aquatic ecosystems. Freshw. Biol. 37, 113-132.

Kändler, M., Blechinger, K., Seidler, C., Pavlů, V., Šanda, M., Dostál, T., Krása, J., Vitvar, T., Štich, M., 2017. Impact of land use on water quality in the upper Nisa catchment in the Czech Republic and in Germany. Sci. Total Environ. 586, 1316-1325.

Karr, J.R., Schlosser, I.J., 1978. Water resources and the land-water interface. Science 201, 229-234.

Keesstra, S.D., Kondrlova, E., Czajka, A., Seeger, M., Maroulis, J., 2012. Assessing riparian zone impacts on water and sediment movement: a new approach. Neth. J. Geosci. 91 (1-2), 245-255.

Lessels, J.S., Bishop, T.F.A., 2013. Estimating water quality using linear mixed models with stream discharge and turbidity. J. Hydrol. 498, 13-22.

Lima, P.R.A., 2003. Balanço de espécies químicas em microbacias sob utilização agrícola região de Botucatu/SP. Master thesisFaculdade de Ciências Agronômicas, Universidade Estadual Paulista, Botucatu, Brazil (in Portuguese).

Lima, P.R.A., 2013. Balanço de espécies químicas em microbacias sob utilização agrícola região de Botucatu/SP [tese]. Faculdade de Ciências Agronômicas, Universidade Estadual Paulista, Botucatu.

Lorion, C.M., Kennedy, B.P., 2009. Relationships between deforestation, riparian forest buffers and benthic macroinvertebrates in neotropical headwater streams. Freshw. Biol. 54 (1), 165-180.

MacDonald, L.H., Coe, D., 2007. Influence of headwater streams on downstream reaches in forested areas. For. Sci. 53, 148-168.

Malmer, A., Grip, H., 1989. Soil disturbance and loss of infiltrability caused by mechanized and manual extraction of tropical rainforest in Sabah, Malaysia. For. Ecol. Manag. 38 $1-12$.

Markewitz, D., Resende, J.C.F., Parron, L., Bustamante, M., Klink, C.A., Figuereido, R.O. Davidson, E.A., 2006. Dissolved rainfall inputs and streamwater outputs in an undisturbed watershed on highly weathered soils in the Brazilian cerrado. Hydrol. Process 20, 2615-2639.

Masselink, R.J., Heckmann, T., Temme, A.J., Anders, N.S., Gooren, H., Keesstra, S.D., 2017a. A network theory approach for a better understanding of overland flow connectivity. Hydrol. Process. 31 (1), 207-220.

Masselink, R., Temme, A.J.A.M., Gimenez, R., Casali, J., Keesstra, S.D., 2017b. Assessing hillslope-channel connectivity in an agricultural catchment using rare-earth oxide tracers and random forests models. Cuadernos de Investigacion Geografica 43, 19-39.

McClain, M.E., Naiman, R.J., 2008. Andean influences on the biogeochemistry and ecology of the Amazon River. Bioscience 58 (4), 325-338.

Osborne, L.L., Kovacic, D.A., 1993. Riparian vegetated buffer strips in water-quality restoration and stream management. Freshw. Biol. 29, 243-258.

Parron, L.M., Bustamante, M.M.C., Markewitz, D., 2010. Fluxes of nitrogen and phosphorus in a gallery forest in the Cerrado of central Brazil. Biogeochemistry 105, 89-104.

Parsons, A.J., Bracken, L., Poeppl, R.E., Wainwright, J., Keesstra, S.D., 2015. Introduction to special issue on connectivity in water and sediment dynamics. Earth Surf. Process. Landf. 40 (9), 1275-1277.

Perez Hernandez, D., Lopez, J.L., 1998. Alguno aspectos relevantes de la hidrologia del Rio Orinoco. In: Lopez Sanchez, J.L., Saavedra Cuadra, I.I., Dubois Martinez, M. (Eds.), E Rio Orinoco. Aprovechamiento Sustentable. Memorias de las primeras jornadas venezolanas de investigacion sobre el Rio Orinoco. Instituto de Mecanica de Fluidos, Facultad de Ingenieria, Universidad Central de Venezuela, Caracas, pp. 138-154, 16 e 20 noviembre de 1998 (in Spanish).

Peterjohn, W.T., Correll, D.L., 1984. Nutrient dynamics in an agricultural watershed: observations on the role of a riparian forest. Ecology 65, 1466-1475.

Pinto, L.V.A., 2003. Caracterização física da bacia do ribeirão Santa Cruz, Lavras, MG, e propostas de recuperação de suas nascentes. Master thesisUniversidade Federal de Lavras, Brazil (in Portuguese).

Primavesi, O., Freitas, A.R., Primavesi, A.C., Oliveira, H.T., 2002. Water quality of the Canchim's creek watershed in São Carlos, SP, Brasil, occupied by beef and dairy cattle activities. Braz. Arch. Biol. Technol. 45 (2), 209-218. 
Pringle, C.M., Scatena, F.N., 1999. Aquatic ecosystem deterioration in Latin America and the Caribbean. In: Hatch, L.U., Swisher, M.E. (Eds.), Managed Ecosystems: The Mesoamerican Experience. Oxford University Press, New York, pp. 104-113.

Richardson, J.S., Danehy, R.J., 2007. A synthesis of the ecology of headwater streams and their riparian zones in temperate forests. For. Sci. 53, 131-147.

Rocha, J., Hughes, S.J., Almeida, P., Garcia-Cabral, I., Amich, F., Crespí, A.L., 2015. Contemporary and future distribution patterns of fluvial vegetation under different climate change scenarios and implications for integrated water resource management. Ecol. Res. 30 (6), 989-1003.

Rodrigues, V., Estrany, J., Ranzini, M., Cicco, V., Martin-Benito, M.J., Hedo, J., Lucas-Borja M.E., 2018. Effects of land use and seasonality on stream water quality in a small tropical catchment: the headwater of Corrego Agua Limpa, Sao Paulo (Brazil). Sci. Total Environ. 622-623, 1553-1561.

Schumm, S.A., 1977. The Fluvial System. Wiley and Sons, New York.

Sener, S., Sener, E., Davraz, A., 2017. Evaluation of water quality using water quality index (WQI) method and GIS in Aksu River (SW-Turkey). Sci. Total Environ. 584, 131-144.

Shrestha, S., Kazama, F., 2007. Assessment of surface water quality using multivariate statistical techniques: a case study of the Fuji river basin, Japan. Environ. Model. Softw. 22, 464-475.

Silva, J.S.O., Cunha-Bustamante, M.M., Markewitz, D., Krusche, A.V., Ferreira, L.G., 2011. Effects of land cover on chemical characteristics of streams in the Cerrado region of Brazil. Biogeochemistry 105, 75-88.

Singh, P., Haritashya, U.K., Kumar, N., 2004. Seasonal changes in meltwater storage and drainage characteristics of the Dokriani Glacier, Garhwal Himalayas (India). Hydrol. Res. 35 (1), 15-29.

Slattery, M.C., Gares, P.A., Phillips, J.D., 2002. Slope-channel linkage and sediment delivery on North Carolina coastal plain cropland. Earth Surf. Process. Landf. 27, 1377-1387.

Smart, R.P., Soulsby, C., Neal, C., Wade, A., Cresser, M.S., Billett, M.F., Langan, S.J., Edwards, A.C., Jarvie, H.P., Owen, R., 1998. Factors regulating the spatial and temporal distribution of solute concentrations in a major river system in NE Scotland. Sci. Total Environ. $221,93-110$.
Soulsby, C., Langan, S.J., Neal, C., 2001. Environmental change, land use and water quality in Scotland: current issues and future prospects. Sci. Total Environ. 265, 387-394.

Spaans, E.J.A., Bouma, J., Lansu, A., Wielemaker, W.G., 1990. Measuring soil hydraulic properties after clearing of tropical rain forest in a Costa Rican soil. Trop. Agric. (Trinidad) 67, 61-65.

Strahler, A.N., 1952. Hypsometric (area-altitude) analysis of erosional topography. Bull. Geol. Soc. Am. 63 (11), 1117-1142.

Tabacchi, E. Lambs, L. Guilloy, H., Planty-Tabacchi, A.M Muller, E. Decamps, H., 2000. Impacts of riparian vegetation on hydrological processes. Hydrol. Process. 14 (16-17), 2959-2976.

Viswanathan, V.C., Molson, J., Schirmer, M., 2015. Does river restoration affect diurnal and seasonal changes to surface water quality? A study along the Thur River, Switzerland. Sci. Total Environ. 532, 91-102.

Vuori, K.-M., 1995. Direct and indirect effects of iron on river ecosystems. Ann. Zool. Fenn. 32, 317-329.

Wear, D.N., Turner, M.G., Naiman, R.J., 1998. Land cover along an urban-rural gradient: implications for water quality. Ecol. Appl. 8 (3), 619-630.

Wohl, E., 2017. The significance of small streams. Front. Earth Sci. 1-10.

Xia, L.L., Liu, R.Z., Zao, Y.W., 2012. Correlation analysis of landscape pattern and water quality in Baiyangdian watershed. Procedia Environ Sci 13, 2188-2196.

Ye, Y., He, X., Chen, W., Yao, J., Yu, S., Jia, L., 2014. Seasonal water quality upstream of Dahuofang Reservoir, China - the effects of land use type at various spatial scales. Clean (Weinh) 42, 1423-1432.

Yu, S., Xu, Z., Wu, W., Zuo, D., 2016. Effect of land use types on stream water quality under seasonal variation and topographic characteristics in the Wei River basin, China. Ecol. Indic. 60, 202-212.

Zhou, T., Wu, J., Peng, S., 2012. Assessing the effects of landscape pattern on river water quality at multiple scales: a case study of the Dongjiang River watershed, China. Ecol. Indic. 23, 166-175. 